\title{
The 6D Bias and the Equity-Premium Puzzle
}

\section{Citation}

Gabaix, Xavier, and David Laibson. 2001. "The 6D Bias and the Equity-Premium Puzzle." NBER Macroeconomics Annual 16 (January): 257-312. https://doi.org/10.1086/654447.

\section{Permanent link}

http://nrs.harvard.edu/urn-3:HUL.InstRepos:41467450

\section{Terms of Use}

This article was downloaded from Harvard University's DASH repository, and is made available under the terms and conditions applicable to Other Posted Material, as set forth at http:// nrs.harvard.edu/urn-3:HUL.InstRepos:dash.current.terms-of-use\#LAA

\section{Share Your Story}

The Harvard community has made this article openly available.

Please share how this access benefits you. Submit a story.

Accessibility 
This PDF is a selection from a published volume from the National Bureau of Economic Research

Volume Title: NBER Macroeconomics Annual 2001, Volume 16

Volume Author/Editor: Ben S. Bernanke and Kenneth Rogoff, editors

Volume Publisher: MIT Press

Volume ISBN: 0-262-02520-5

Volume URL: http://www.nber.org/books/bern02-1

Conference Date: April 20-21, 2001

Publication Date: January 2002

Title: The 6D Bias and the Equity-Premium Puzzle Author: Xavier Gabaix, David Laibson

URL: http://www.nber.org/chapters/c11067 


\section{Xavier Gabaix and David Laibson}

MIT; and Harvard University and NBER

\section{The $6 \mathrm{D}$ Bias and the Equity-Premium Puzzle}

\section{Introduction}

Consumption growth covaries only weakly with equity returns, which seems to imply that equities are not very risky. However, investors have historically received a very large premium for holding equities. For twenty years, economists have asked why an asset with little apparent risk has such a large required return. ${ }^{1}$

Grossman and Laroque (1990) argued that adjustment costs might answer the equity-premium puzzle. If it is costly to change consumption, households will not respond instantaneously to changes in asset prices. Instead, consumption will adjust with a lag, explaining why consumption growth covaries only weakly with current equity returns. In Grossman and Laroque's framework, equities are risky, but that riskiness does not show up in a high contemporaneous correlation between consumption growth and equity returns. The comovement is only observable in the long run.

Lynch (1996) and Marshall and Parekh (1999) have simulated discretetime delayed-adjustment models and demonstrated that these models can potentially explain the equity-premium puzzle. ${ }^{2}$ In light of the complexity of these models, both sets of authors used numerical simulations.

We thank Ben Bernanke, Olivier Blanchard, John Campbell, James Choi, Karen Dynan, George Constantinides, John Heaton, Robert Lucas, Anthony Lynch, Greg Mankiw, Jonathan Parker, Monika Piazzesi, Ken Rogoff, James Stock, Jaume Ventura, Annette Vissing, and seminar participants at Delta, Insead, Harvard, MIT, University of Michigan, NBER, and NYU for helpful comments. We thank Emir Kamenica, Guillermo Moloche, Eddie Nikolova, and Rebecca Thornton for outstanding research assistance.

1. For the intellectual history of this puzzle, see Rubinstein (1976), Lucas (1978), Shiller (1982), Hansen and Singleton (1983), Mehra and Prescott (1985), and Hansen and Jagannathan (1991). For useful reviews see Kocherlakota (1996) and Campbell (1999).

2. See also related work by Caballero (1995), He and Modest (1995), Heaton and Lucas (1996), Luttmer (1995), and Lynch and Balduzzi (2000). 
We propose a continuous-time generalization of Lynch's (1996) model. Our extension provides two new sets of results. First, our analysis is analytically tractable; we derive a complete analytic characterization of the model's dynamic properties. Second, our continuous-time framework generates effects that are up to six times larger than those in discrete-time models.

We analyze an economy composed of consumers who update their consumption every $D$ (as in "delay") periods. Such delays may be motivated by decision costs, attention allocation costs, and/or mental accounts. $^{3}$ The core of the paper describes the consequences of such delays. In addition, we derive a sensible value of $D$ based on a decisioncost framework.

The $6 D$ bias is our key result. Using data from our economy, an econometrician estimating the coefficient of relative risk aversion (CRRA) from the consumption Euler equation would generate a multiplicative CRRA bias of $6 D$. For example, if agents adjust their consumption every $D=4$ quarters, and the econometrician uses quarterly aggregates in his analysis, the imputed coefficient of relative risk aversion will be 24 times greater than the true value. Once we take account of this $6 D$ bias, the Euler-equation tests are unable to reject the standard consumption model. High equity returns and associated violations of the HansenJagannathan (1991) bounds cease to be puzzles.

The basic intuition for this result is quite simple. If households adjust their consumption every $D \geq 1$ periods, then on average only $1 / D$ households will adjust each period. Consider only the households that adjust during the current period, and assume that these households adjust consumption at dates spread uniformly over the period. Normalize the timing so the current period is the time interval $[0,1]$. When a household adjusts at time $i \in[0,1]$, it can only respond to equity returns that have already been realized by time $i$. Hence, the household can only respond to fraction $i$ of within-period equity returns. Moreover, the household that adjusts at time $i$ can only change consumption for the remainder of the period. Hence, only a fraction $1-i$ of this period's consumption is affected by the change at time $i$. On average the households that adjust during the current period display a covariance between equity returns and consumption growth that is biased down by factor

$\int_{0}^{1} i(1-i) d i=\frac{1}{6}$

3. See Gabaix and Laibson (2000b) for a discussion of decision costs and attention allocation costs. See Thaler (1992) for a discussion of mental accounts. 
The integral is taken from 0 to 1 to average over the uniformly distributed adjustment times.

Since only a fraction $1 / D$ of households adjust in the first place, the aggregate covariance between equity returns and consumption growth is approximately $\frac{1}{6} \times 1 / D$ as large as it would be if all households adjusted instantaneously. The Euler equation for the instantaneous-adjustment model implies that the coefficient of relative risk aversion is inversely related to the covariance between equity returns and consumption growth. If an econometrician used this Euler equation to impute the coefficient of relative risk aversion, and he used data from our delayed adjustment economy, he would impute a coefficient of relative risk aversion that was $6 D$ times too large.

In Section 2 we describe our formal model, motivate our assumptions, and present our key analytic finding. In Section 2.2 we provide a heuristic proof of our results for the case $D \geq 1$. In Section 3 we present additional results that characterize the dynamic properties of our model economy. In Section 4 we close our framework by describing how $D$ is chosen. In Section 5 we consider the consequences of our model for macroeconomics and finance. In Section 6 we discuss empirical evidence that supports the Lynch (1996) model and our generalization. The model matches most of the empirical moments of aggregate consumption and equity returns, including a new test which confirms the $6 D$ prediction that the covariance between $\ln \left(C_{t+h} / C_{t}\right)$ and $R_{t+1}$ should slowly rise with $h$. In Section 7 we conclude.

\section{Model and Key Result}

Our framework is a synthesis of ideas from the continuous-time model of Merton (1969) and the discrete-time model of Lynch (1996). In essence we adopt Merton's continuous-time modeling approach and Lynch's emphasis on delayed adjustment. ${ }^{4}$

We assume that the economy has two linear production technologies: a risk-free technology and a risky technology (i.e., equities). The risk-free technology has instantaneous return $r$. The returns from the risky technology follow a geometric diffusion process with expected return $r+\pi$ and standard deviation $\sigma$.

We assume that consumers hold two accounts: a checking account and a balanced mutual fund. A consumer's checking account is used for day-to-day consumption, and this account holds only the risk-free asset.

4. See Calvo (1983), Fischer (1977), and Taylor (1979) for earlier examples of delayed adjustment in macroeconomics. 
The mutual fund is used to replenish the checking account from time to time. The mutual fund is professionally managed and is continuously rebalanced so that a share $\theta$ of the mutual-fund assets is always invested in the risky asset. ${ }^{5}$ The consumer is able to pick $\theta \cdot{ }^{6}$ In practice, the consumer picks a mutual fund that maintains the consumer's preferred value of $\theta$. We call $\theta$ the equity share (in the mutual fund).

Every $D$ periods, the consumer looks at her mutual fund and decides how much wealth to withdraw from it to deposit in her checking account. Between withdrawal periods-i.e., from withdrawal date $t$ to the next withdrawal date $t+D$-the consumer spends from her checking account and does not monitor her mutual fund. For now we take $D$ to be exogenous. Following a conceptual approach taken in Duffie and Sun (1990), we later calibrate $D$ with a decision-cost model (see Section 4). Alternatively, $D$ can be motivated with a mental-accounting model of the type proposed by Thaler (1992).

Finally, we assume that consumers have isoelastic preferences and exponential discount functions:

$U_{i t}=E_{t} \int_{s=t}^{\infty} e^{-\rho(s-t)}\left(\frac{c_{i s}^{1-\gamma}-1}{1-\gamma}\right) d s$.

Here $i$ indexes the individual consumer and $t$ indexes time.

We adopt the following notation. Let $w_{i t}$ represent the wealth in the mutual fund at date $t$. Between withdrawal dates, $w_{i t}$ evolves according to $d w_{i t}=w_{i t}\left[(r+\theta \pi) d t+\theta \sigma d z_{t}\right]$

where $z_{t}$ is a Wiener process. We can now characterize the optimal choices of our consumer. We describe each date at which the consumer monitors-and in equilibrium withdraws from-her mutual fund as a reset date. Formal proofs of all results are provided in the appendix.

PROPOSITION 1 On the equilibrium path, the following properties hold:

1. Between reset dates, consumption grows at a fixed rate $(1 / \gamma)(r-\rho)$.

2. The balance in the checking account just after a reset date equals the net present value (NPV) of consumption between reset dates, where the NPV is taken with the risk-free rate.

5. This assumption can be relaxed without significantly changing the quantitative results. In particular, the consumer could buy assets in separate accounts without any instantaneous rebalancing.

6. The fact that $\theta$ does not vary once it is chosen is optimal from the perspective of the consumer in this model. 
3. At reset date $\tau$, consumption is $c_{i \tau^{+}}=\alpha w_{i \tau^{-}}$, where $\alpha$ is a function of the technology parameters, preference parameters, and $D$.

4. The equity share in the mutual fund is

$\theta=\frac{\pi}{\gamma \sigma^{2}}$

Here $c_{i \tau^{+}}$represents consumption immediately after reset, and $w_{i \tau^{-}}$represents wealth in the mutual fund immediately before reset.

Claim 1 follows from the property that between reset dates the rate of return to marginal savings is fixed and equal to $r$. So between reset dates the consumption path grows at the rate derived in Ramsey's (1928) original deterministic growth model:

$\frac{\dot{c}}{c}=\frac{1}{\gamma}(r-\rho)$.

Claim 2 reflects the advantages of holding wealth in the balanced mutual fund. Instantaneous rebalancing of this fund makes it optimal to store "extra" wealth-i.e., wealth that is not needed for consumption between now and the next reset date-in the mutual fund. So the checking account is exhausted between reset dates. Claim 3 follows from the homotheticity of preferences. Claim 4 implies that the equity share is equal to the same equity share derived by Merton (1969) in his instantaneous-adjustment model. This exact equivalence is special to our institutional assumptions, but approximate equivalence is a general property of models of delayed adjustment (see Rogers, 2001, for numerical examples in a related model). Note that the equity share is increasing in the equity premium $(\pi)$ and decreasing in the coefficient of relative risk aversion $(\gamma)$ and the variance of equity returns $\left(\sigma^{2}\right)$.

Combining claims 1-3 implies that the optimal consumption path between date $\tau$ and date $\tau+D$ is $c_{i t}=\alpha e^{(1 / \gamma)(r-\rho)(t-\tau)} w_{i \tau^{-}}$and the optimal balance in the checking account just after reset date $\tau$ is

$\int_{\tau}^{\tau+D} c_{i s} e^{-r(s-\tau)} d s=\int_{\tau}^{\tau+D} \alpha e^{(1 / \gamma)(r-\rho)(s-\tau)-r(s-\tau)} w_{i \tau}-d s$.

Claim 3 implies that at reset dates optimal consumption is linear in wealth. The actual value of the propensity to consume, $\alpha$, does not matter for the results that follow. Any linear rule-e.g., linear rules of thumb- 
will suffice. In practice, the optimal value of $\alpha$ in our model will be close to the optimal marginal propensity to consume derived by Merton,

$\alpha=\frac{\rho}{\gamma}+\left(1-\frac{1}{\gamma}\right)\left(r+\frac{\pi^{2}}{2 \gamma \sigma^{2}}\right)$.

Merton's value is exactly optimal in our framework when $D=0$.

\subsection{OUR KEY RESULT: THE $6 D$ BIAS}

In our economy, each agent resets consumption at intervals of $D$ units of time. Agents are indexed by their reset time $i \in[0, D)$. Agent $i$ resets consumption at dates $\{i, i+D, i+2 D, \ldots\}$.

We assume that the consumption reset times are distributed uniformly. ${ }^{7}$ More formally, there exists a continuum of consumers whose reset indexes $i$ are distributed uniformly over $[0, D)$. So the proportion of agents resetting their consumption in any time interval of length $\Delta t \leq D$ is $\Delta t / D$.

To fix ideas, suppose that the unit of time is a quarter of the calendar year, and $D=4$. In other words, the span of time from $t$ to $t+1$ is one quarter of a year. Since $D=4$, each consumer will adjust her consumption once every four quarters. We will often choose the slightly nonintuitive normalization that a quarter of the calendar year is one period, since quarterly data constitute the natural unit of temporal aggregation with contemporary macroeconomic data.

Call $C_{t}$ the aggregate consumption between $t-1$ and $t$ :

$C_{t}=\int_{i=0}^{D}\left(\int_{s=t-1}^{t} c_{i s} d s\right) \frac{1}{D} d i$

Note that $\int_{s=t-1}^{t} c_{i s} d s$ is per-period consumption for consumer $i$.

Suppose that an econometrician estimates $\gamma$ and $\beta$ using a consumption Euler equation (i.e., the consumption CAPM). What will the econometrician infer about preferences?

THEOREM 2 Consider an economy with true coefficient of relative risk aversion $\gamma$. Suppose an econometrician estimates the Euler equation

$E_{t-1}\left[\hat{\beta}\left(\frac{C_{t}}{C_{t-1}}\right)^{-\hat{\gamma}} R_{t}^{a}\right]=1$

7. The results change only a little when we relax the assumption of a uniform distribution. Most importantly, if reset dates were clumped at the end of periods-a natural assumption - then the implied bias would be infinite. 
for two assets: the risk-free bond and the stock market. In other words, the econometrician fits $\hat{\beta}$ and $\hat{\gamma}$ to match the Euler equation above for both assets. Then the econometrician will find

$$
\hat{\gamma}=\left\{\begin{array}{lll}
6 D \gamma & \text { for } & D \geq 1 \\
\frac{6}{3(1-D)+D^{2}} \gamma & \text { for } & 0 \leq D \leq 1
\end{array}\right.
$$

plus higher-order terms characterized in subsequent sections.

Figure 1 plots $\hat{\gamma} / \gamma$ as a function of $D$. The formulae for the cases $0 \leq D$ $\leq 1$ and $D \geq 1$ are taken from Theorem 2 .

The two formulae paste at the crossover point, $D=1$. Convexity of the formula below $D=1$ implies that $\hat{\gamma} / \gamma \geq 6 D$ for all values of $D$. The case of instantaneous adjustment (i.e., $D=0$ ) is of immediate interest, since it has been solved already by Grossman, Melino, and Shiller (1987). With $D=0$ the only bias arises from time aggregation of the econometrician's data, not delayed adjustment by consumers. Grossman, Melino, and Shiller show that time aggregation produces a bias of $\hat{\gamma} / \gamma=2$, matching our formula for $D=0$.

The most important result is the equation for $D \geq 1, \hat{\gamma}=6 D \gamma$, which we call the $6 D$ bias. For example, if each period $(t$ to $t+1)$ is a quarter of a calendar year, and consumption is reset every $D=4$ quarters, then we

\section{FIGURE 1 RATIO OF ESTIMATED $\hat{\gamma}$ TO TRUE $\gamma$}

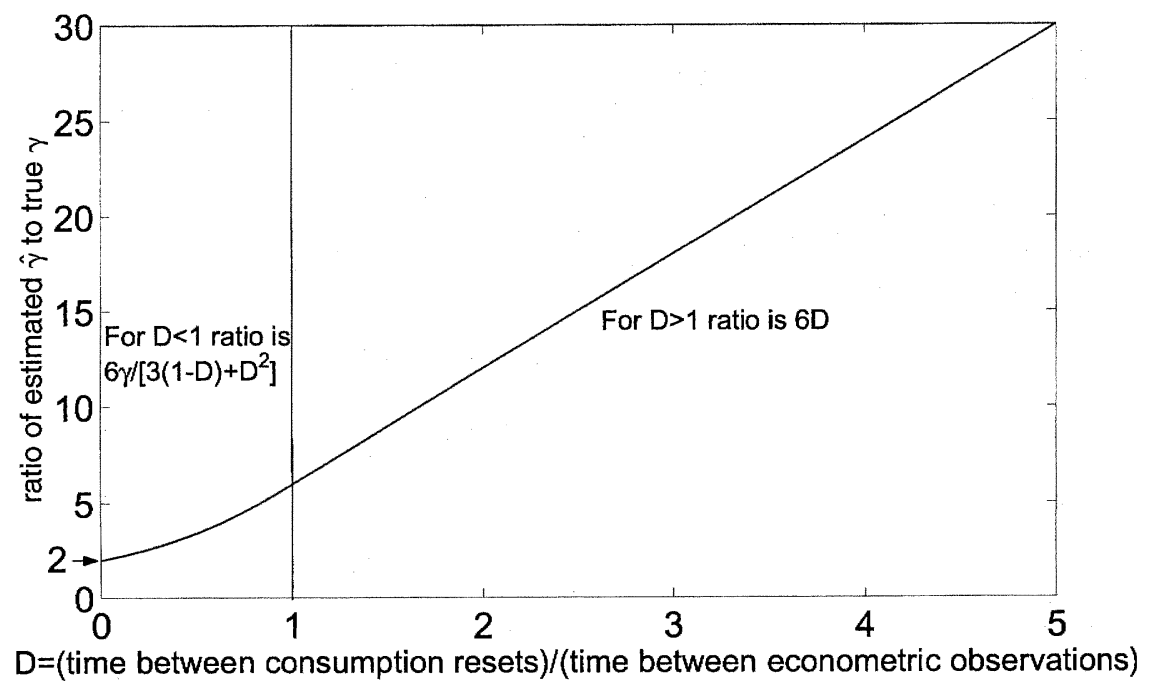


get $\hat{\gamma}=24 \gamma$. Hence $\gamma$ is overestimated by a factor of 24 . If consumption is revised every 5 years, then we have $D=20$, and $\hat{\gamma}=120 \gamma$.

Reset periods of 4 quarters or more are not unreasonable in practice. For an extreme case, consider the 30-year-old employee who accumulates balances in a retirement savings account [e.g., a 401(k)] and fails to recognize any fungibility between these assets and his preretirement consumption. In this case, stock-market returns will affect consumption at a considerable lag ( $D>120$ quarters for this example).

However, such extreme cases are not necessary for the points that we wish to make. Even with a delay of only 4 quarters, the implications for the equity-premium puzzle literature are dramatic. With a multiplicative bias of 24, econometrically imputed coefficients of relative risk aversion of 50 suddenly appear quite reasonable, since they imply actual coefficients of relative risk aversion of roughly 2 .

In addition, our results do not rely on the strong assumption that all reset rules are time- and not state-contingent. In Appendix B we incorporate the realistic assumption that all households adjust immediately when the equity market experiences a large (Poisson) shock. In practice, such occasional state-contingent adjustments only slightly modify our results.

Our qualitative results are robust to our assumption about the uniform distribution of adjustment dates. For example, if adjustment occurs at the end (or beginning) of the quarter, then the multiplicative bias in the estimated coefficient of relative risk aversion is infinite, since the continuous flow of consumption in the current quarter is unaffected by current asset returns. By contrast, if adjustments occur at exactly the middle of the quarter, then the multiplicative bias is $4 \mathrm{D}$, since the consumers that do adjust can only respond to half of the stock returns and their adjustment only affects half of the consumption flow (i.e. $1 / 2 * 1 / 2=1 / 4$ ).

We can also compare the $6 \mathrm{D}$ bias analytically with the biases that Lynch (1996) simulates numerically in his original discrete-time model. In Lynch's framework, agents consume every month and adjust their portfolio every $T$ months. Lynch's econometric observation period is the union of $F$ one-month intervals, so $D=T / F$. In Appendix $C$ we show that when $D \geq 1$ Lynch's framework generates a bias which is bounded below by $D$ and bounded above by $6 D$. Specifically, an econometrician who naively estimated the Euler equation with data from Lynch's economy would find a bias of

$$
\frac{\hat{\gamma}}{\gamma}=D \frac{6 F^{2}}{(F+1)(F+2)}+\text { higher-order terms. }
$$


Holding $D$ constant, the continuous-time limit corresponds to $F \rightarrow \infty$, and for this case $\hat{\gamma} \gamma=6 D$. The discrete-time case where agents consume at every econometric period corresponds to $F=1$, implying $\hat{\gamma} / \gamma=D$, which can be derived directly.

Finally, the $6 D$ bias complements participation bias (e.g., Vissing, 2000; Brav, Constantinides, and Geczy, 2000). If only a fraction $s$ of agents hold a significant share of their wealth in equities (say $s=\frac{1}{3}$ ), then the covariance between aggregate consumption and returns is lower by a factor $s$. As Theorem 8 demonstrates, this bias combines multiplicatively with our bias: if there is limited participation, the econometrician will find the values of $\hat{\gamma}$ in Theorem 2, divided by s. In particular, for $D \geq 1$, he will find

$\hat{\gamma}=\frac{6 D}{s} \gamma$

This formula puts together three important biases generated by Eulerequation (and Hansen-Jagannathan) tests: $\hat{\gamma}$ will be overestimated because of time aggregation and delayed adjustment (the $6 D$ factor), and because of limited participation (the $1 / \mathrm{s}$ factor).

\subsection{ARGUMENT FOR $D \geq 1$}

In this section we present a heuristic proof of Theorem 2. A rigorous proof is provided in Appendix A.

Normalize a generic period to be one unit of time. The econometrician observes the return of the stock market from 0 to 1 :

$\ln R_{1}=r+\pi-\frac{\sigma^{2}}{2}+\sigma \int_{0}^{1} d z_{s}$

where $r$ is the risk-free interest rate, $\pi$ is the equity premium, $\sigma^{2}$ is the variance of stock returns, and $z$ is a Wiener process. The econometrician also observes aggregate consumption over the period:

$C_{1}=\int_{i=0}^{D}\left(\int_{s=0}^{1} c_{i s} d s\right) \frac{1}{D} d i$

As is well known, when returns and consumption are assumed to be jointly lognormal, the standard Euler equation implies that ${ }^{8}$

8. $E_{t-1}\left[\hat{\beta}\left(C_{t} / C_{t-1}\right)^{-\hat{\gamma}} R_{t}^{a}\right]=1$ with $R_{t}^{a}=e^{\mu_{a}-\sigma_{a}^{2} / 2+\sigma_{a} \varepsilon_{a}}$. The subscripts and superscripts $a$ denote asset-specific returns and standard deviations. As Hansen and Singleton (1983) showed, 


$$
\hat{\gamma}=\frac{\pi}{\operatorname{cov}\left(\ln \frac{C_{1}}{C_{0}}, \ln R_{1}\right)} .
$$

We will show that when $D \geq 1$ the measured covariance between consumption growth and stock-market returns, $\operatorname{cov}\left(\ln \left[C_{1} / C_{0}\right], \ln R_{1}\right)$, will be lower by a factor $6 D$ than the instantaneous covariance, $\operatorname{cov}\left(d \ln C_{t}, d\right.$ $\left.\ln R_{t}\right) / d t$, that arises in the frictionless CCAPM. As is well known, in the frictionless CCAPM

$$
\gamma=\frac{\pi}{\operatorname{cov}\left(d \ln C_{t}, d \ln R_{t}\right) / d t} .
$$

Assume that each agent consumes one unit in period $[-1,0] .{ }^{9}$ So aggregate consumption in period $[-1,0]$ is also one: $C_{0}=1$. Since $\ln \left(C_{1} / C_{0}\right) \simeq$ $C_{1} / C_{0}-1$, we can write

$$
\begin{aligned}
\operatorname{cov}\left(\ln \frac{C_{1}}{C_{0}}, \ln R_{1}\right) & \simeq \operatorname{cov}\left(C_{1}, \ln R_{1}\right) \\
& =\int_{0}^{D} \operatorname{cov}\left(C_{i 1}, \ln R_{1}\right) \frac{1}{D} d i
\end{aligned}
$$

with $C_{i 1}=\int_{0}^{1} c_{i s} d s$ the time-aggregated consumption of agent $i$ during period $[0,1]$.

First, take the case $D=1$. Agent $i \in[0,1)$ changes her consumption at time $i$. For $s \in[0, i)$, she has consumption $c_{i s}=\alpha w_{i \tau}-e^{(1 / \gamma)(r-\rho)(s-\tau)}$, where $\tau=i-D$.

Throughout this paper we use approximations to get analytic results. Let $\varepsilon \equiv \max \left(r, \rho, \theta \pi, \sigma^{2}, \sigma^{2} \theta^{2}, \alpha\right)$. When we use annual periods, $\varepsilon$ will be

$$
\ln \hat{\beta}+\mu_{a}-\hat{\gamma}\left(\mu_{c}-\frac{\sigma_{c}^{2}}{2}+\hat{\gamma} \frac{\sigma_{c}^{2}}{2}\right)-\hat{\gamma} \sigma_{a c}=0 .
$$

If we evaluate this expression for the risk-free asset and equities, we find that

$$
\pi=\hat{\gamma} \operatorname{cov}\left(\ln \frac{C_{t}}{C_{t-1}}, \ln R_{t}\right) .
$$

Note that $\pi+r=\mu_{a}$.

9. This assumption need not hold exactly. Consumption need be unity only up to $O_{<0}(\sqrt{\varepsilon})+O(\varepsilon)$ terms, in the notation defined below. 
approximately $0.05 .{ }^{10}$ For quarterly periods, $\varepsilon$ will be approximately 0.01 . We can express our approximation errors in higher-order terms of $\varepsilon$.

Since consumption in period $[-1,0]$ is normalized to one, at time $\tau=$ $i-D, \alpha$ times wealth will be equal to 1 plus small corrective terms; more formally,

$\alpha w_{i \tau^{-}}=1+O_{<0}(\sqrt{\varepsilon})+O(\varepsilon)$,

$\alpha w_{i \tau^{+}}=1+O_{<0}(\sqrt{\varepsilon})+O(\varepsilon)$.

Here $O(\varepsilon)$ represents stochastic or deterministic terms of order $\varepsilon$, and $O_{<0}(\sqrt{\varepsilon})$ represents stochastic terms that depend only on equity innovations that happen before time 0 . Hence the $O_{<0}(\sqrt{\varepsilon})$ terms are all orthogonal to equity innovations during period $[0,1]$.

Drawing together our last two results, for $s \in[0, i)$,

$$
\begin{aligned}
c_{i s} & =e^{(1 / \gamma)(r-\rho)(s-\tau)} \alpha w_{i \tau^{-}} \\
& =[1+O(\varepsilon)]\left[1+O_{<0}(\sqrt{\varepsilon})+O(\varepsilon)\right] \\
& =1+O_{<0}(\sqrt{\varepsilon})+O(\varepsilon) .
\end{aligned}
$$

Without loss of generality, set $z(0)=0$. So consumer $i$ 's mutual fund wealth at date $t=i^{-}$is

$$
\begin{aligned}
\alpha w_{i, t=i^{-}} & =e^{\left(r+\theta \pi-\theta^{2} \sigma^{2} / 2 D+\theta \sigma[z(i)-z(i-D)]\right.} \alpha w_{i \tau^{+}} \\
& =\left[1+\theta \sigma z(i)+O_{<0}(\sqrt{\varepsilon})+O(\varepsilon)\right]\left[1+O_{<0}(\sqrt{\varepsilon})+O(\varepsilon)\right] \\
& =1+\theta \sigma z(i)+O_{<0}(\sqrt{\varepsilon})+O(\varepsilon)
\end{aligned}
$$

The consumer adjusts consumption at $t=i$, and so for $s \in[i, 1]$ she consumes

$$
\begin{aligned}
c_{i s} & =e^{(1 / \gamma)(r-\rho)(s-i)} \alpha w_{i, t=i^{-}} \\
& =[1+O(\varepsilon)]\left[1+\theta \sigma z(i)+O_{<0}(\sqrt{\varepsilon})+O(\varepsilon)\right] \\
& =1+\theta \sigma z(i)+O_{<0}(\sqrt{\varepsilon})+O(\varepsilon) .
\end{aligned}
$$

The covariance of consumption and returns for agent $i$ is

10. For a typical annual calibration $r=0.01, \rho=0.05, \theta \pi=(0.78)(0.06), \sigma^{2}=(0.16)^{2}$, $\sigma^{2} \theta^{2}=(\pi / \gamma \sigma)^{2}=(0.06 / 3 \times 0.16)^{2}$, and $\alpha=0.04$. 


$$
\begin{aligned}
\operatorname{cov}\left(C_{i 1}, \ln R_{1}\right)= & \int_{0}^{1} \operatorname{cov}\left(c_{i s}, \ln R_{1}\right) d s \\
= & \int_{0}^{i} 0 d s+\int_{i}^{1} \operatorname{cov}\left(1+\theta \sigma z(i)+O_{<0}(\sqrt{\varepsilon})\right. \\
& \left.+O(\varepsilon), \sigma z(1)+r+\pi-\frac{\sigma^{2}}{2}\right) d s \\
= & \int_{i}^{1}\left[\theta \sigma^{2} \operatorname{cov}(z(i), \sigma z(1))+O\left(\varepsilon^{3 / 2}\right)\right] d s \\
& =\theta \sigma^{2} i(1-i)+O\left(\varepsilon^{3 / 2}\right) \\
& \simeq \theta \sigma^{2} i(1-i) .
\end{aligned}
$$

Here and below $\simeq$ means "plus higher-order terms in $\varepsilon . "$

The covariance contains the multiplicative factor $i$ because the consumption change reflects only return information which is revealed between date 0 and date $i$. The covariance contains the multiplicative factor $1-i$ because the change in consumption occurs at time $i$, and therefore affects consumption for only the subinterval $[i, 1]$.

We often analyze "normalized" variances and covariances. Specifically, we divide the moments predicted by the $6 D$ model by the moments predicted by the benchmark model with instantaneous adjustment and instantaneous measurement. Such normalizations highlight the "biases" introduced by the $6 D$ economy.

For the case $D=1$, the normalized covariance of aggregate consumption growth and equity returns is

$$
\begin{aligned}
\frac{1}{\theta \sigma^{2}} \operatorname{cov}\left(C_{1}, \ln R_{1}\right) & =\int_{i=0}^{1} \frac{1}{\theta \sigma^{2}} \operatorname{cov}\left(C_{i 1}, R_{1}\right) \frac{d i}{D} \\
& \simeq \int_{0}^{1} i(1-i) d i=\frac{1}{6}
\end{aligned}
$$

which is the (reciprocal of the) $6 D$ factor for $D=1$.

Consider now the case $D \geq 1$. Consumer $i \in[0, D)$ resets her consumption at $t=i$. During period 1 (i.e., $t \in[0,1]$ ) only agents with $i \in[0,1]$ will reset their consumption. Consumers with $i \in(1, D]$ will not change their consumption, so they will have a zero covariance, $\operatorname{cov}\left(C_{i 1}, R_{1}\right)=0$. Hence, 


$$
\frac{1}{\theta \sigma^{2}} \operatorname{cov}\left(C_{i 1}, R_{1}\right) \simeq \begin{cases}i(1-i) & \text { if } i \in[0,1] \\ 0 & \text { if } i \in[1, D]\end{cases}
$$

For $D \geq 1$ the covariance of aggregate consumption is just $1 / D$ times what it would be if we had $D=1$ :

$$
\begin{aligned}
\frac{1}{\theta \sigma^{2}} \operatorname{cov}\left(\ln \left(C_{1} / C_{0}\right), R_{1}\right) & \simeq \int_{0}^{D} \frac{1}{\theta \sigma^{2}} \operatorname{cov}\left(C_{i 1}, R_{1}\right) \frac{d i}{\mathrm{D}} \\
& =\frac{1}{D} \int_{0}^{1} \frac{1}{\theta \sigma^{2}} \operatorname{cov}\left(C_{i 1}, R_{1}\right) d i \\
& \simeq \frac{1}{D} \int_{0}^{1} i(1-i) d i \\
& =\frac{1}{6 D} .
\end{aligned}
$$

The $6 D$ lower covariance of consumption with returns translates into a $6 D$ higher measured CRRA $\hat{\gamma}$. Since $\theta=\pi / \gamma \sigma^{2}$ [equation (1)], we get

$\operatorname{cov}\left(\ln \frac{C_{1}}{C_{0}}, \ln R_{1}\right)=\frac{\pi}{6 D \gamma}$.

The Euler equation (6) then implies

$\hat{\gamma}=6 D \gamma$,

as anticipated.

Several properties of our result should be emphasized. First, holding $D$ fixed, the bias in $\hat{\gamma}$ does not depend on either preferences or technology: $r, \pi, \sigma, \rho, \gamma$. This independence property will apply to all of the additional results that we report in subsequent sections. When $D$ is endogenously derived, $D$ itself will depend on the preference and technology parameters.

For simplicity, the derivation above assumes that agents with different adjustment indexes $i$ have the same "baseline" wealth at the start of each period. In the long run this wealth equivalence will not apply exactly. However, if the wealth disparity is moderate, the reasoning above will 
still hold approximately. ${ }^{11}$ Numerical analysis with 50 -year adult lives implies that the actual bias is very close to $6 D$, the value it would have if all of the wealth levels were identical period by period.

\section{General Characterization of the Economy}

In this section we provide a general characterization of the dynamic properties of the economy described above. We analyze four properties of our economy: excess smoothness of consumption growth, positive autocorrelation of consumption growth, low covariance of consumption growth and asset returns, and nonzero covariance of consumption growth and lagged equity returns.

Our analysis focuses on first-order effects with respect to the parameters $r, \rho, \theta \pi, \sigma^{2}, \sigma^{2} \theta^{2}$, and $\alpha$. Call $\varepsilon \equiv \max \left(r, \rho, \theta \pi, \sigma^{2}, \sigma^{2} \theta^{2}, \alpha\right)$. We assume $\varepsilon$ to be small. Empirically, $\varepsilon \simeq 0.05$ with a period length of a year, and $\varepsilon \simeq 0.01$ with a period length of a calendar quarter. All the results that follow (except one ${ }^{12}$ ) are proved with $O\left(\varepsilon^{3 / 2}\right)$ residuals. In fact, at the cost of more tedious calculations, one can show that the residuals are actually $O\left(\varepsilon^{2}\right) \cdot{ }^{13}$

The following theorem is the basis of this section. The proof appears in Appendix A.

THEOREM 3 The autocovariance of consumption growth at horizon $h \geq 0$ can be expressed as

$\operatorname{cov}\left(\ln \frac{C_{h+t}}{C_{h+t-1}}, \ln \frac{C_{t}}{C_{t-1}}\right)=\theta^{2} \sigma^{2} \Gamma(D, h)+O\left(\varepsilon^{3 / 2}\right)$,

where

$\Gamma(D, h) \equiv \frac{1}{D^{2}}[d(D+h)+d(D-h)-d(h)-d(-h)]$,

11. More precisely, it is only important that the average wealth of households that switch on date $t$ not differ significantly from the average wealth of households that switch on any date $s \in[t-D, t+D]$. To guarantee this cross-date average similarity we could assume that each reset interval ends stochastically. This randomness generates "mixing" between populations of households that begin life with different reset dates.

12. Equation (12) is proved to $O(\sqrt{\varepsilon})$, but with more tedious calculations can be shown to be $O(\varepsilon)$.

13. One follows exactly the lines of the proofs presented here, but includes higher-order terms. Calculations are available from the authors upon request. 


$$
d(D) \equiv \sum_{i=0}^{4}\left(\begin{array}{c}
4 \\
i
\end{array}\right) \frac{(-1)^{i}}{2 \times 5 !}|D+i-2|^{5},
$$

and $\left(\begin{array}{l}4 \\ i\end{array}\right)=4 / i !(4-i) !$ is the binomial coefficient.

The expressions above are valid for noninteger values of $D$ and $h$. The functions $d(D)$ and $\Gamma(D, h)$ have the following properties, many of which will be exploited in the analysis that follows ${ }^{14}$ :

$d \in C^{4}$.

$d(D)=|D| / 2$ for $|D| \geq 2$.

$d(0)=\frac{7}{30}$.

$\Gamma(D, h) \sim 1 / D$ for large $D$.

$\Gamma(D, h) \geq 0$.

$\Gamma(D, h)>0$ iff $D+2>h$.

$\Gamma(D, h)$ is nonincreasing in $h$.

$\Gamma(D, 0)$ is decreasing in $D$, but $\Gamma(D, h)$ is hump-shaped for $h>0$.

$\Gamma(0, h)=0$ for $h \geq 2$.

$\Gamma(0,0)=\frac{2}{3}$.

$\Gamma(0,1)=\frac{1}{6}$.

Figure 2 plots $d(D)$ along with a second function which we will use below.

\section{$3.1 \Gamma(D, 0)$}

We begin by studying the implications of the autocovariance function, $\Gamma(D, h)$, for the volatility of consumption growth (i.e., by setting $h=0$ ). Like Caballero (1995), we also show that delayed adjustment induces excess smoothness. Corollary 4 describes our quantitative result.

COROLLARY 4 In the frictionless economy $(D=0), \operatorname{var}\left(d C_{t} / C_{t}\right) / d t=\sigma^{2} \theta^{2}$. In our economy, with delayed adjustment and time aggregation bias,

$$
\frac{\operatorname{var}\left(\ln \left[C_{t} / C_{t-1}\right]\right)}{\sigma^{2} \theta^{2}}=\Gamma(D, 0) \leq \frac{2}{3} .
$$

The volatility of consumption, $\sigma^{2} \theta^{2} \Gamma(D, 0)$, decreases as $D$ increases.

The normalized variance of consumption, $\Gamma(D, 0)$, is plotted against $D$ in Figure 3.

14. $\Gamma$ is continuous, so $\Gamma(0, h)$ is intended as $\lim _{D \rightarrow 0} \Gamma(D, h)$. 
FIGURE 2 THE FUNCTIONS $d(x)$ AND $e(x)$

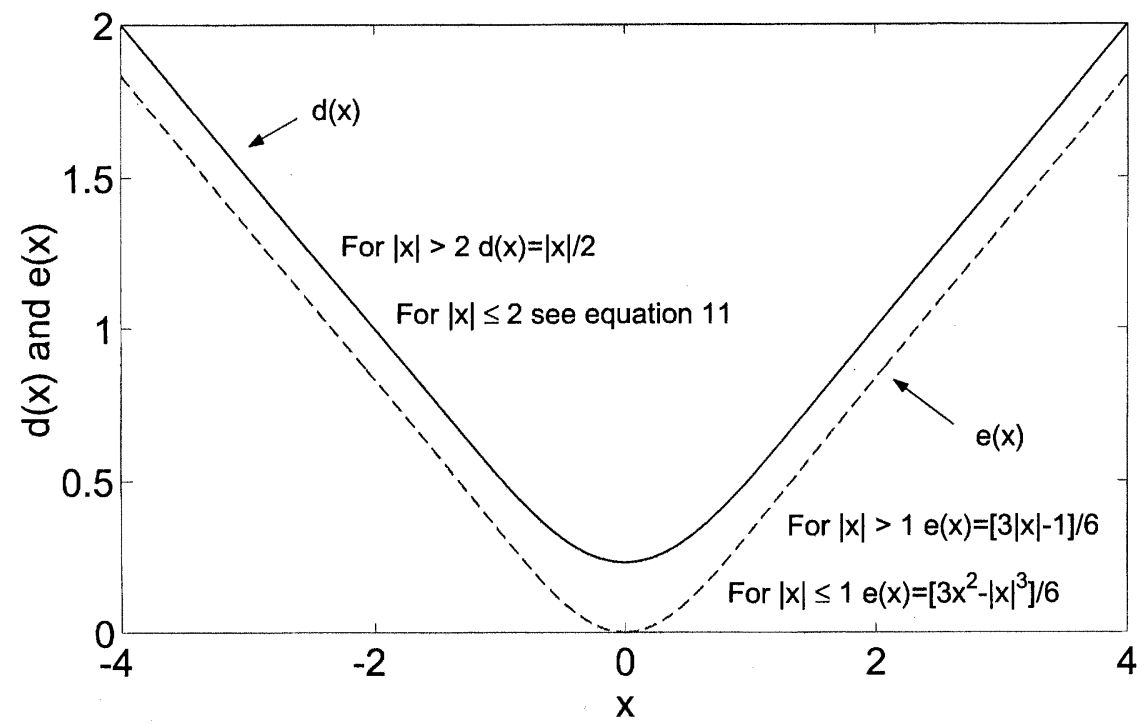

FIGURE 3 THE NORMALIZED VARIANCE OF CONSUMPTION GROWTH, $\Gamma(D, 0)$




For $D=0$, the normalized variance is $\frac{2}{3}$, well below the benchmark value of 1 . The $D=0$ case reflects the bias generated by time aggregation effects. As $D$ rises above zero, delayed adjustment effects also appear. For $D=0,1,2,4,20$ the normalized variance takes values $0.67,0.55$, $0.38,0.22$, and 0.04 . For large $D$, the bias is approximately $1 / D$.

Intuitively, as $D$ increases, none of the short-run volatility of the economy is reflected in consumption growth, since only a proportion $1 / D$ of the agents adjust consumption in any single period. Moreover, the size of the adjustments only grows as $\sqrt{D}$. So the total magnitude of adjustment is falling as $1 / \sqrt{D}$, and the variance falls as $1 / D$.

\section{$3.2 \Gamma(D, h)$ WITH $h>0$}

We now consider the properties of the (normalized) autocovariance function $\Gamma(D, h)$ for $h=1,2,4,8$. Figure 4 plots these respective curves, ordered from $h=1$ on top to $h=8$ at the bottom. Note that in the benchmark case-instantaneous adjustment and no time-aggregation bias - the autocovariance of consumption growth is zero. With only time-aggregation effects, the one-period autocovariance is $\Gamma(0,1)=\frac{1}{6}$, and all $h$-period autocovariances with $h>1$ are zero.

FIGURE 4 NORMALIZED AUTOCOVARIANCE $\Gamma(D, h)$ WITH $h=1,2,4,8$

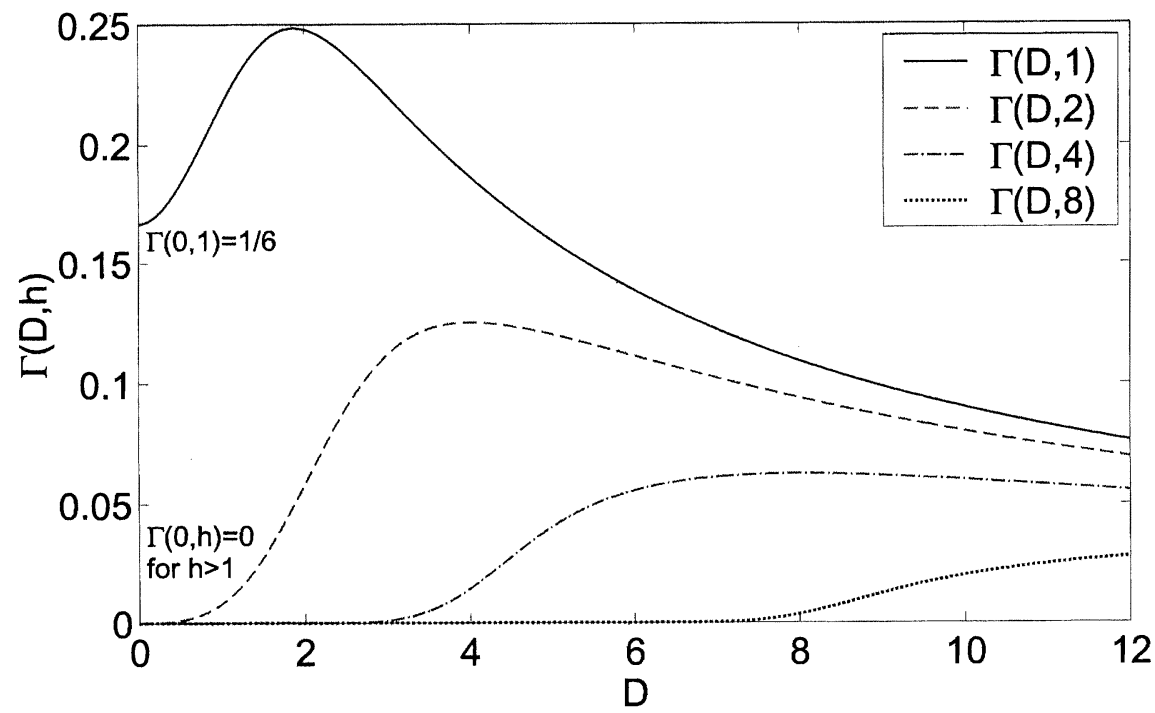




\subsection{REVISITING THE EQUITY-PREMIUM PUZZLE}

We can also state a formal and more general analogue of Theorem 2 .

PROPOSITION 5 Suppose that consumers reset their consumption every $h_{a}$ periods. Then the covariance between consumption growth and stock-market returns at horizon $h$ will be

$\operatorname{cov}\left(\ln \frac{C_{[t, t+h]}}{C_{[t-h, t]}}, \ln R_{[t, t+h]}\right)=\frac{\theta \sigma^{2} h}{b(D)}+O\left(\varepsilon^{3 / 2}\right)$,

where $D=h_{a} / h$ and

$b(D)=\left\{\begin{array}{lll}6 D & \text { for } & D \geq 1, \\ \frac{6}{3(1-D)+D^{2}} & \text { for } & 0 \leq D \leq 1 .\end{array}\right.$

The associated correlation is

$\operatorname{corr}\left(\ln \frac{C_{[t, t+h]}}{C_{[t-h, t]}}, \ln R_{[t, t+h]}\right)=\frac{1}{b(D) \Gamma(D, 0)^{1 / 2}}+O\left(\varepsilon^{1 / 2}\right)$

In the benchmark model with continuous sampling and adjustment, the covariance is just

$\frac{\operatorname{cov}\left(d \ln C_{t}, d \ln R_{t}\right)}{d t}=\theta \sigma^{2}$

Moreover, in that model the covariance at horizon $h$ is just

$\operatorname{cov}\left(\ln \frac{C_{[t, t+h]}}{C_{[t-h, t]}}, \ln R_{[t, t+h]}\right)=\theta \sigma^{2} h$

So the effect introduced by the $6 D$ model is captured by the factor $1 / b(D)$ which appears in Proposition 5.

We compare this benchmark with the effects generated by our discrete-observation, delayed-adjustment model. As the horizon $h$ tends to $+\infty$, the normalized covariance between consumption growth and asset returns tends to

$\lim _{h \rightarrow \infty} \frac{\theta \sigma^{2} h}{b\left(h_{a} / h\right)} \frac{1}{\theta \sigma^{2} h}=\frac{1}{b(0)}=\frac{1}{2}$, 
which is true for any fixed value of $h_{a}$. This effect is due exclusively to time aggregation. Delayed adjustment ceases to matter as the horizon length goes to infinity.

Proposition 5 covers the special case discussed in Section 2: horizon $h=1$, and reset period $h_{a}=D \geq 1$. For this case, the normalized covariance is approximately equal to

$\frac{\theta \sigma^{2}}{b(D)} \frac{1}{\theta \sigma^{2}}=\frac{1}{6 D}$

Figure 5 plots the multiplicative covariance bias factor $1 / b\left(h_{a} / h\right)$ as a function of $h$, for $h_{a}=1$. In the benchmark case (i.e., continuous sampling and instantaneous adjustment) there is no bias; the bias factor is unity. In the case with only time-aggregation effects (i.e., discrete sampling and $\left.h_{a}=0\right)$ the bias factor is $1 / b(0 / h)=\frac{1}{2}$.

Hence, low levels of comovement show up most sharply when horizons are low. For $D \geq 1$ (i.e., $h_{a} / h \geq 1$ ), the covariance between consumption growth and stock returns is $6 D$ times lower than one would expect in the model with continuous adjustment and continuous sampling.

FIGURE 5 MULTIPLICATIVE COVARIANCE BIAS FACTOR $1 / b(1 / h)$

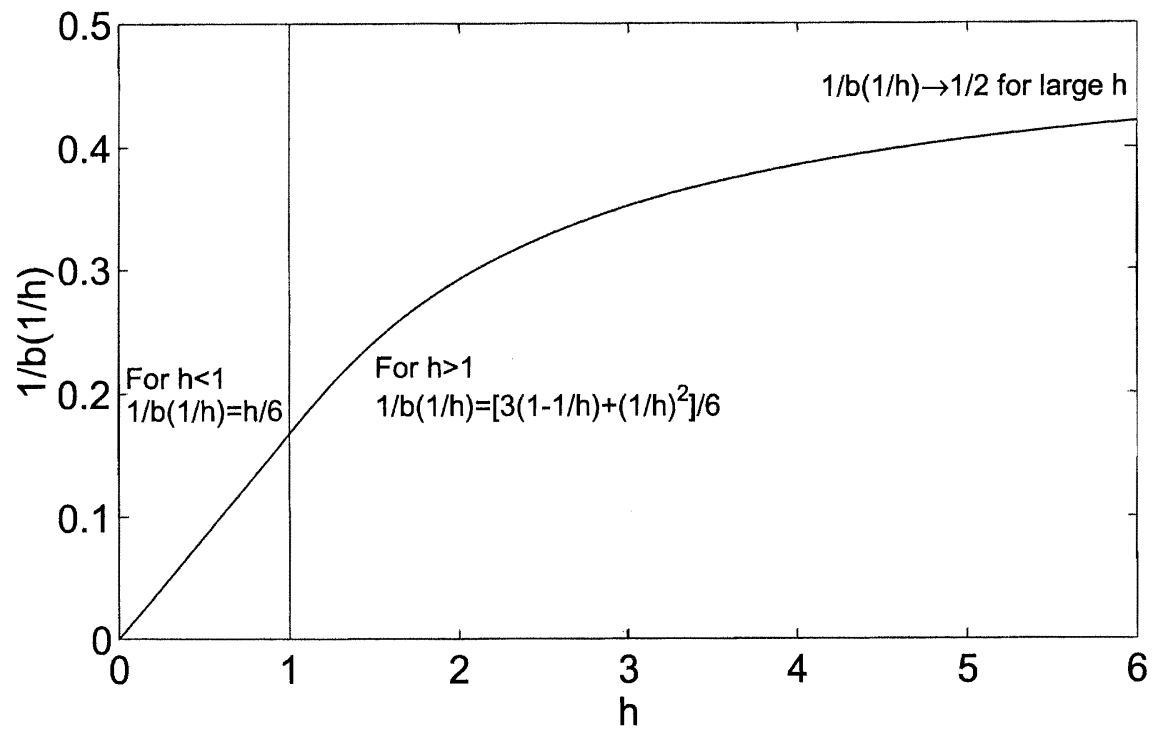


We now characterize the covariance between current consumption growth and lagged equity returns.

THEOREM 6 Suppose that consumers reset their consumption every $h_{a}=D h$ periods. Then the covariance between $\ln \left(C_{[t, t+1]} / C_{[t-1, t]}\right)$ and lagged equity returns $\ln R_{\left[t+s_{1}, t+s_{2}\right]}\left(s_{1}<s_{2} \leq 1\right)$ will be

$\operatorname{cov}\left(\ln \frac{C_{[t, t+1]}}{C_{[t-1, t]}}, \ln R_{\left[t+s_{1}, t+s_{2}\right]}\right)=\theta \sigma^{2} V\left(D, s_{1}, s_{2}\right)+O\left(\varepsilon^{3 / 2}\right)$

with

$V\left(D, s_{1}, s_{2}\right)=\frac{e\left(s_{1}\right)-e\left(s_{2}\right)-e\left(s_{1}+D\right)+e\left(s_{2}+D\right)}{D}$,

where

$e(x)=\left\{\begin{array}{lll}\frac{3 x^{2}-|x|^{3}}{6} & \text { for } & |x| \leq 1, \\ \frac{3|x|-1}{6} & \text { for } & |x| \geq 1 .\end{array}\right.$

The following corollary will be used in the empirical section.

COROLLARY 7 The covariance between $\ln \left(C_{[s+h-1, s+h]} / C_{[s-1, s]}\right)$ and lagged equity returns $\ln R_{[s, s+1]}$ will be

$$
\begin{aligned}
\operatorname{cov}( & \left(\ln \frac{C_{[s+h-1, s+h]}}{C_{[s-1, s]}}, \ln R_{[s, s+1]}\right) \\
= & \theta \sigma^{2} \frac{e(1+D)-e(1)-e(1-h+D)+e(1-h)}{D}+O\left(\varepsilon^{3 / 2}\right) .
\end{aligned}
$$

In particular, when $h \geq D+2, \operatorname{cov}\left(\ln \left[C_{[s+h-1, s+h]} / C_{[s-1, s]}\right], \ln R_{[s, s+1]}\right)=\theta \sigma^{2}$; one sees full adjustment at horizons (weakly) greater than $D+2$.

In practice, Theorem 6 is most naturally applied when the lagged equity returns correspond to specific lagged time periods: $s_{2}=s_{1}+1, s_{1}=$ $0,-1,-2, \ldots$ 


\section{FIGURE 6 NORMALIZED COVARIANCE OF CONSUMPTION \\ GROWTH AND LAGGED ASSET RETURNS, $V(D, s, s+1)$, FOR $D=0.25,1,2,4$}

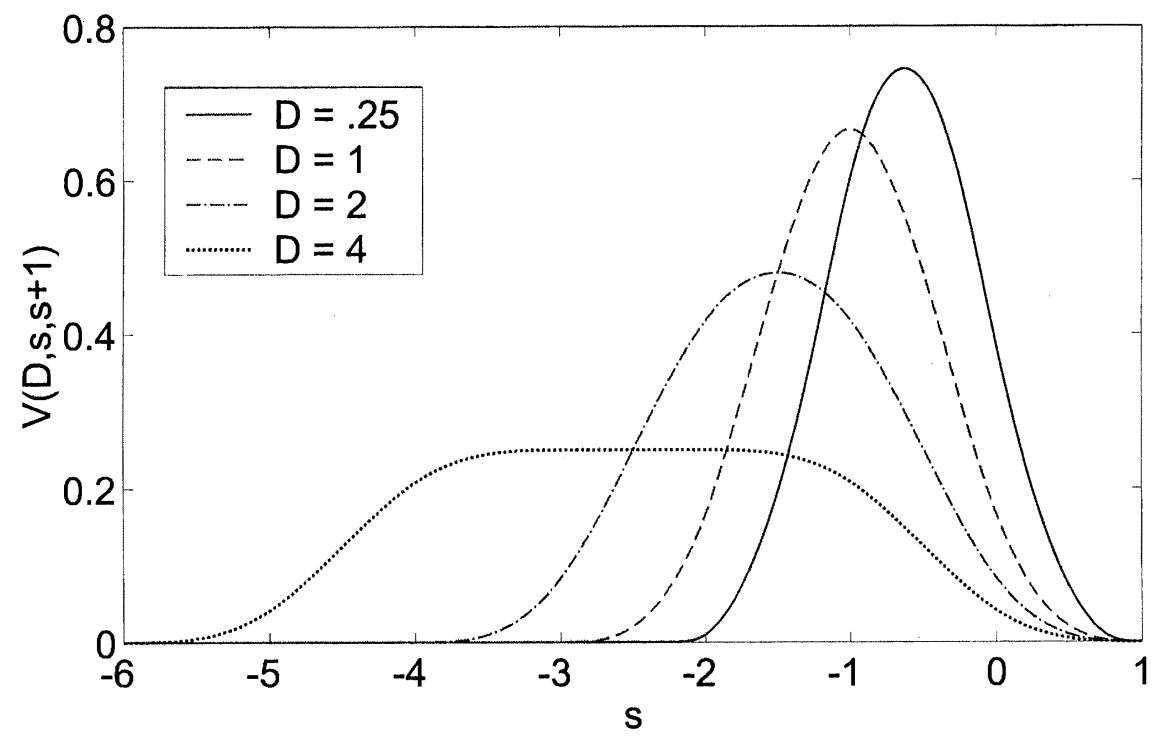

Note that $V\left(D, s_{1}, s_{2}\right)>0$ iff $s_{2}>-D-1$. Hence, the covariance in Theorem 6 is positive only at lags 0 through $D+1$.

Figure 6 plots the normalized covariances of consumption growth and lagged asset returns for different values of $D$. Specifically, we plot $V(D, s, s+1)$ against $s$ for $D=0.25,1,2,4$, from right to left.

Consider a regression of consumption growth on some arbitrary (large) number of lagged returns,

$\ln \frac{C_{t+1}}{C_{t}}=\sum_{s=\underline{s}}^{0} \beta_{s} \ln R_{t+1+s}$

One should find

$\beta_{s}=\theta V(D, s, s+1)$

Note that the sum of the normalized lagged covariances is one:

$\frac{1}{\theta \sigma^{2}} \sum_{s=-\infty}^{0} \operatorname{cov}\left(\ln \frac{C_{[t, t+1]}}{C_{[t-1, t]}} \ln R_{[t+s, t+s+1]}\right)=\sum_{s=-\infty}^{0} V(D, s, s+1)=1$. 
This implies that the sum of the coefficients will equal the portfolio share of the stock market, 15

$$
\sum_{s=-D-1}^{0} \beta_{s}=\theta
$$

\subsection{EXTENSION TO MULTIPLE ASSETS AND HETEROGENEITY IN $D$}

We now extend the framework to the empirically relevant case of multiple assets with stochastic returns. We also introduce heterogeneity in $D$ 's. Such heterogeneity may arise because different $D$ 's apply to different asset classes and because $D$ may vary across consumers.

Say that there are different types of consumers $l=1, \ldots, n_{l}$ and different types of asset accounts $m=1, \ldots, n_{m}$. Consumers of type $l$ exist in proportion $p_{l}\left(\Sigma_{l} p_{l}=1\right)$ and look at account $m$ every $D_{l m}$ periods. The consumer has wealth $w_{l m}$ invested in account $m$, and has an associated marginal propensity to consume (MPC), $\alpha_{l m}$. In most models the MPC's will be the same for all assets, but for the sake of behavioral realism and generality we consider possibly different MPC's.

For instance, income shocks could have a low $D=1$, stock-market shocks a higher $D=4$, and shocks to housing wealth a $D=40.16$ Account $m$ has standard deviation $\sigma_{m}$ and shocks $d z_{m t}$. Denote by $\rho_{m n}=$ $\operatorname{cov}\left(d z_{n t}, d z_{m t}\right) / d t$ the correlation matrix of the shocks, and by $\sigma_{m n}=\rho_{m n} \sigma_{m} \sigma_{n}$ their covariance matrix.

Total wealth in the economy is $\Sigma_{l, m} p_{l} w_{l m}$ and total consumption $\Sigma_{l, m} p_{l} \alpha_{l m} w_{l m}$. A useful and natural quantity is

$\theta_{l m}=\frac{p_{l} \alpha_{l m} w_{l m}}{\sum_{l^{\prime}, m^{\prime}} p_{l^{\prime}} \alpha_{l^{\prime} m^{\prime}} w_{l^{\prime} m^{\prime}}}$

A shock $d z_{m t}$ in wealth account $m$ will get translated at mean interval $\frac{1}{2} \Sigma_{l} p_{l} D_{l m}$ into a consumption shock $d C / C=\Sigma_{l} \theta_{l m} d z_{m t}$.

We can calculate the second moments of our economy.

15. This is true in a world with only equities and riskless bonds. In general, it's more appropriate to use a model with several assets, including human capital, as in the next section.

16. This example implies different short-run marginal propensities to consume out of wealth windfalls in different asset classes. Thaler (1992) describes one behavioral model with similar asset-specific marginal propensities to consume. 
THEOREM 8 In the economy described above, we have

$\operatorname{cov}\left(\ln \frac{C_{t}}{C_{t-1}}, \ln R_{\left[t+s_{1}, t+s_{2}\right]}^{n}\right)=\sum_{l, m} \theta_{l m} \sigma_{m n} V\left(D_{l m}, s_{1}, s_{2}\right)+O\left(\varepsilon^{3 / 2}\right)$

and

$\operatorname{cov}\left(\ln \frac{C_{t+h}}{C_{t+h-1}}, \ln \frac{C_{t}}{C_{t-1}}\right)=\sum_{l, l^{\prime}, m, m^{\prime}} \theta_{l m} \theta_{l^{\prime} m^{\prime}} \sigma_{m m^{\prime}} \Gamma\left(D_{l m^{\prime}} D_{l^{\prime} m^{\prime}}, h\right)+O\left(\varepsilon^{3 / 2}\right)$

with

$\Gamma\left(D, D^{\prime}, h\right)=\frac{1}{D D^{\prime}}\left[d(\mathrm{D}+\mathrm{h})+d\left(D^{\prime}-h\right)-d\left(D^{\prime}-D-h\right)-d(h)\right]$,

$V$ defined in (14), and defined in (11).

The function $\Gamma(D, t)$, defined earlier in $(10)$, relates to $\Gamma\left(D, D^{\prime}, t\right)$ by $\Gamma(D, D, t)=\Gamma(D, t)$. Recall that $V(D, 0,1)=1 / b(D)$. So a conclusion from (19) is that, when there are several types of people and assets, the bias that the econometrician would find is the harmonic mean of the individual biases $b\left(D_{l m}\right)$, the weights being given by the shares of variance.

As an application, consider the case with identical agents $\left(n_{l}=1 ; l\right.$ is suppressed for this example) and different assets with the same MPC, $\alpha_{m}$ $=\alpha$. Recall that $V(D, 0,1)=1 / b(D)$. So the bias $\hat{\gamma} / \gamma$ will be

$\frac{\hat{\gamma}}{\gamma}=\left(\sum_{m} \frac{\theta_{m}^{2} \sigma_{m}^{2}}{\sum_{m^{\prime}} \theta_{m^{\prime}}^{2} \sigma_{m^{\prime}}^{2}} b\left(D_{m}\right)^{-1}\right)^{-1}$.

Hence, with several assets, the aggregate bias is the weighted mean of the biases, the mean being the harmonic mean, and the weight of asset $m$ being the share of the total variance that comes from this asset. This allows us, in Appendix B, to discuss a modification of the model with differential attention to big shocks (jumps).

These relationships are derived exactly along the lines of the singleasset, single-type economy of the previous sections. Equation (19) is the covariance between returns, $\ln R_{\left[t+s_{1}, t+s_{2}\right]}^{n}=\sigma_{n} z_{\left[t+s_{1}, t+s_{2}\right]}^{n}+O(\varepsilon)$, and the representation formula for aggregate consumption is 
$\ln \frac{C_{t}}{C_{t-1}}=\sum_{m} \theta_{m} \sigma_{m} \int_{-1}^{1} a(i) z_{\left[t-1+i-D_{m}, t-1+i\right]}^{m} d i+O\left(\varepsilon^{3 / 2}\right)$,

where $a(i)=(1-|i|)^{+}$. Equation (23) can also be used to calculate the autocovariance (20) of consumption, if one defines

$\Gamma\left(D, D^{\prime}, h\right)=\int_{i, j \in[-1,1]} a(i) a(j) \operatorname{cov}\left(z_{[t-1+i-D, t-1+i]}, z_{\left[t-1+j+h-D^{\prime}, t-1+j+h\right]}\right) \frac{d i}{D} \frac{d j}{D^{\prime}}$.

The closed-form expression (21) of $\Gamma$ is derived in Appendix A.

\subsection{SKETCH OF THE PROOF}

Proofs of the propositions appear in Appendix A. In this subsection we provide intuition for those arguments. We start with the following representation formula for consumption growth.

PROPOSITION 9 We have

$\ln \frac{C_{t+1}}{C_{t}}=\theta \sigma \int_{-1}^{1} a(i) z_{[t+i-D, t+i]} \frac{1}{D} d i+O(\varepsilon)$.

Note that the order of magnitude of $\theta \sigma \int_{-1}^{1} a(i) z_{[t+i-D, t+i]} d i /_{D}$ is the order of magnitude of $\sigma$, i.e. $O(\sqrt{\varepsilon})$.

Assets returns can be represented as $\ln R_{\left[t+s_{1}, t+s_{2}\right]}=\sigma z_{\left[t+s_{1}, t+s_{2}\right.}+O(\varepsilon)$. So we get

$$
\begin{aligned}
\operatorname{cov}( & \left.\ln \frac{C_{t}}{C_{t-1}}, \ln R_{\left[s+s_{1}, s+s_{2}\right]}\right) \\
= & \theta \sigma^{2} \int_{-1}^{1} a(i) \operatorname{cov}\left(z_{[t-1+i-D, t-1+i]}, z_{\left[s+s_{1}, s+s_{2}\right]}\right) \frac{d i}{D}+O\left(\varepsilon^{3 / 2}\right) \\
= & \theta \sigma^{2} \int_{-1}^{1} a(i) \lambda\left([t-1+i-D, t-1+i] \cap\left[s+s_{1}, s+s_{2}\right]\right) \frac{d i}{D} \\
& +O\left(\varepsilon^{3 / 2}\right) .
\end{aligned}
$$

Here $\lambda(I)$ is the length (the Lebesgue measure) of the interval $I$. Likewise one gets 


$$
\begin{aligned}
\operatorname{cov}( & \left.\ln \frac{C_{h+t}}{C_{h+t-1}}, \ln \frac{C_{t}}{C_{t-1}}\right) \\
= & \theta^{2} \sigma^{2} \int_{-1}^{1} \int_{-1}^{1} a(i) a(j) \operatorname{cov}\left(z_{[h+t-1+i-D, h+t-1+i]}, z_{[t-1+j-D, t-1+j]}\right) \frac{d i}{D} \frac{d j}{D}+O\left(\varepsilon^{3 / 2}\right) \\
= & \theta^{2} \sigma^{2} \int_{-1}^{1} \int_{-1}^{1} a(i) a(j) \lambda([h+t-1+i-D, h+t-1+i] \\
& \cap[t-1+j-D, t-1+j]) \frac{d i}{D} \frac{d j}{D}+O\left(\varepsilon^{3 / 2}\right) .
\end{aligned}
$$

The bulk of the proof is devoted to the explicit calculation of this last equation and equation (27).

\section{Endogenizing $D$}

Until now, we have assumed that $D$ is fixed exogenously. In this section we discuss how $D$ is chosen, and provide a framework for calibrating $D$.

Because of delayed adjustment, the actual consumption path will deviate from the first-best instantaneously adjusted consumption path. In steady state, the welfare loss associated with this deviation is equivalent, using a money metric, to a proportional wealth loss of ${ }^{17}$

$\Lambda_{C}=\frac{\gamma}{2} E\left(\frac{\Delta C}{C}\right)^{2}+$ higher-order terms

Here $\Delta C$ is the difference between actual consumption and first-best instantaneously adjusted consumption. If the asset is observed every $D$ periods, we have

$\Lambda_{C}=\frac{1}{4} \gamma \theta^{2} \sigma^{2} D+O\left(\varepsilon^{2}\right)$.

Equations (28) and (29) are derived in Appendix A. We assume ${ }^{18}$ that each consumption adjustment costs a proportion $q$ of the wealth $w$. A

17. This is a second-order approximation. See Cochrane (1989) for a similar derivation.

18. This would come from a utility function

$$
U=E\left[\left(1-q \sum_{i \geq 0} e^{-\rho \tau_{i}}\right)^{1-\gamma} \int_{0}^{\infty} e^{-\rho s} \frac{c_{i s}^{1-\gamma}}{1-\gamma} d s\right]
$$

if the adjustments to consumption are made at dates $\left(\tau_{i}\right)_{i \geq 0}$. A session of consumption planning at time $t$ lowers utility by a consumption equivalent of $q e^{-\rho t}$. 
sensible calibration of $q$ would be $q w=(1 \%)$ (annual consumption) $=$ $(0.01)(0.04) w=\left(4 \times 10^{-4}\right) w$.

The NPV of costs as a fraction of current wealth is $q \sum_{n \geq 0} e^{-\rho n D}$, implying a total cognitive cost of

$$
\Lambda_{q}=\frac{q}{1-e^{-\rho D}}
$$

The optimal $D$ minimizes both consumption variability costs and cognitive costs, i.e., $D^{*}=\arg \min \Lambda_{C}+\Lambda_{q}$ :

$D^{*}=\arg \min _{D} \frac{1}{4} \gamma \theta^{2} \sigma^{2} D+\frac{q}{1-e^{-\rho D}}$

so

$$
\begin{aligned}
\frac{1}{4} \gamma \theta^{2} \sigma^{2} & =q \rho \frac{e^{-\rho D}}{\left(1-e^{-\rho D}\right)^{2}}=\frac{q \rho}{\left(e^{\rho D / 2}-e^{-\rho D / 2}\right)^{2}} \\
& =\frac{q \rho}{4 \sinh ^{2} \frac{\rho D}{2}},
\end{aligned}
$$

and we find for the optimal $D$

$$
\begin{aligned}
D^{*} & =\frac{2}{\rho} \operatorname{arcsinh} \sqrt{\frac{q \rho}{\gamma \theta^{2} \sigma^{2}}} \\
& \simeq \frac{2}{\theta \sigma} \sqrt{\frac{q}{\gamma \rho}}
\end{aligned}
$$

when $\rho D<<1$.

We make the following calibration choices: $q=4 \times 10^{-4}, \sigma^{2}=(0.16)^{2}$, $\gamma=3, \rho=0.01, \pi=0.06$, and $\theta=\pi /\left(\gamma \sigma^{2}\right)=0.78$. Substituting into our equation for $D$, we find

$D \simeq 2$ years.

This calibration implies that $D$-values of at least 1 year (or 4 quarters) are quite easy to defend. Moreover, our formula for $D^{*}$ is highly sensitive to the value of $\theta$. If a liquidity-constrained consumer has only a small 
fraction of her wealth in equities-because most of her wealth is in other forms like human capital or home equity-then the value of $D$ will be quite large. If $\theta=0.05$ because of liquidity constraints, then $D^{*} \simeq 30$ years.

Note that formula (30) would work for other types of shocks than stock-market shocks. With several accounts indexed by $m$, people would pay attention to account $m$ at intervals of length

$D_{m}=\frac{2}{\rho} \operatorname{arcsinh} \sqrt{\frac{q_{m} \rho}{\gamma \theta_{m}^{2} \sigma_{m}^{2}}}$

with $q_{m} w_{m}$ representing the cost of evaluating asset $m$, and $\theta_{m}$ generalized as in equation (18). Equation (31) implies sensible comparative statics on the frequency of reappraisal. Thus we get a mini-theory of the allocation of attention across accounts. ${ }^{19}$

\section{Consequences for Macroeconomics and Finance}

\subsection{SIMPLE CALIBRATED MACRO MODEL}

To draw together the most important implications of this paper, we describe a simple model of the U.S. economy. We use our model to predict the variability of consumption growth, the autocorrelation of consumption growth, and the covariance of consumption growth with equity returns.

Assume the economy is composed of two classes of consumers: stockholders and nonstockholders. ${ }^{20}$ The consumers that we model in Section 2 are stockholders. Nonstockholders do not have any equity holdings, and instead consume earnings from human capital. Stockholders have aggregate wealth $S_{t}$, and nonstockholders have aggregate wealth $N_{t}$. Total consumption is given by the weighted sum

$C_{t}=\alpha\left(S_{t}+N_{t}\right)$

Recall that $\alpha$ is the marginal propensity to consume. So consumption growth can be decomposed into

19. See Gabaix and Laibson $(2000 a, b)$ for a broader theoretical and empirical analysis of attention allocation.

20. This is at a given point in time. A major reason for nonparticipation is that relatively young agents have most of their wealth in human capital, against which they cannot borrow to invest in equities (see Constantinides, Donaldson, and Mehra, 2000). 
$\frac{d C}{C}=\frac{s d S}{S}+\frac{n d N}{N}$

Here $s$ represents the wealth of stockholders divided by the total wealth of the economy, and $n=1-s$ represents the wealth of nonstockholders divided by the total wealth of the economy. So $s$ and $n$ are wealth shares for stockholders and nonstockholders respectively. We make the simplifying approximation that $s$ and $n$ are constant in the empirically relevant medium run.

Using a first-order approximation,

$\ln \left(C_{t} / C_{t-1}\right)=s \ln \left(S_{t} / S_{t-1}\right)+n \ln \left(N_{t} / N_{t-1}\right)$.

If stockholders have loading in stocks $\theta$, the ratio of stock wealth to total wealth in the economy is

$\Theta=s \theta$.

To calibrate the economy we begin with the observation that human capital claims about $\frac{2}{3}$ of GDP $Y$. In this model, human capital is the discounted net present value of labor income accruing to the current cohort of nonstockholders. We assume that the expected duration of the remaining working life of a typical worker is 30 years, implying that the human capital of the current workforce is equal to

$H=\int_{0}^{30} e^{-r t} \frac{2}{3} Y d t=\frac{2\left(1-e^{-30 r}\right)}{3 r} Y \simeq 17 Y$

where $Y$ is aggregate income. Capital income claims $\frac{1}{3}$ of GDP. Assuming that it has the riskiness (and the returns) of the stock market, the amount of capital is

$K=\frac{1}{3(r+\pi)} Y \simeq 5 Y$,

so that the equity share of total wealth is

$\Theta=\frac{K}{K+H} \simeq 0.22$ 
By assuming that all capital is identical to stock-market capital, we implicitly increase the predicted covariance between stock returns and consumption growth. A more realistic model would assume a more heterogeneous capital stock, and hence a lower covariance between stock returns and consumption growth.

In this model economy, we work with data at the quarterly frequency. We assume $\sigma=0.16 / \sqrt{4}, \pi=0.06 / 4, r=0.01 / 4$, and $\gamma=3$, so the equity share [equation (1) above] is $\theta=\pi /\left(\gamma \sigma^{2}\right)=0.78$. Then equation (32) implies $s=0.28$. In other words, $28 \%$ of the wealth in this economy is owned by shareholders. All of stockholders' claims are in either stock or risk-free bonds. To keep things simple, we counterfactually assume that $N$ and $S$ are uncorrelated.

We have to take a stand on the distribution of $D^{\prime}$ s in the economy. We assume that $D$-values are uniformly distributed from 0 to $\bar{D}=120$ quarters (i.e., 30 years). We adopt this distribution to capture a wide range of investment styles. Extremely active investors will have a $D$-value close to 0 , while passive savers may put their retirement wealth in a special mental account, effectively ignoring the accumulating wealth until after age 65 (Thaler, 1992). We are agnostic about the true distribution of $D$ types, and we present this example for illustrative purposes. Any wide range of $D$-values would serve to make our key points.

To keep the focus on stockholders, we assume that nonstockholders adjust their consumption instantaneously in response to innovations in labor income-i.e., at intervals of length 0 .

Theorem 3 implies that the quarterly volatility of aggregate consumption growth is

$\sigma_{C}^{2}=n^{2} \Gamma(0,0) \sigma_{N}^{2}+\Theta^{2} \sigma^{2} \iint_{D, D^{\prime} \in[0, \bar{D}]} \Gamma\left(D, D^{\prime}, 0\right) \frac{d D d D^{\prime}}{\bar{D}^{2}}$.

We assume that the quarterly standard deviation of growth in human capital is $\sigma_{N}=0.01 . .^{21}$ Our assumptions jointly imply that $\sigma_{C}=0.0063 .{ }^{22}$ Most of this volatility comes from variation in the consumption of nonstockholders. Stockholders generate relatively little consumption vola-

21. We calibrate $\sigma_{N}$ from postwar U.S. data on wage growth. From 1959 to 2000 the standard deviation of per capita real wage growth at the quarterly frequency has been 0.0097 (National Income and Product Accounts, Commerce Department, Bureau of Economic Analysis). If wages follow a random walk, then the standard deviation of growth in human capital, $\sigma_{N}$, will equal the standard deviation in wage growth.

22. Figure 3 plots the function $\Gamma(D, 0)$. Note that $\Gamma(0,0)=\frac{2}{3}$ and that $\Gamma(D, 0) \simeq 1 / D$ for large $D$. In the decomposition of $\sigma_{C}^{2}$ above, $n^{2} \Gamma(0,0) \sigma_{N}^{2}=0.34 \times 10^{-4}$ and $\Theta^{2} \sigma^{2} \iint_{D, D^{\prime} \in[0, \bar{D}]} \Gamma\left(D, D^{\prime}, 0\right) d D d D^{\prime} / \bar{D}^{2}=0.049 \times 10^{-4}$. 
tility, because they represent a relatively small share of total consumption and because they only adjust consumption every $D$ periods. This adjustment rule smooths out the response to wealth innovations, since only a fraction $1 / D$ of stockholders adjust their consumption during any single period and the average adjustment is of magnitude $\sqrt{D}$.

Our model's implied quarterly consumption volatility $-\sigma_{\mathrm{C}}=0.0063-$ lies below its empirical counterpart. We calculate the empirical $\sigma_{C}$ using the cross-country panel dataset created by Campbell (1999). ${ }^{23}$ We estimate $\sigma_{C}=0.0106$ by averaging across all of the countries in Campbell's dataset: Australia, Canada, France, Germany, Italy, Japan, the Netherlands, Spain, Sweden, Switzerland, the United Kingdom, and the United States. ${ }^{24}$ Part of the gap between our theoretical standard deviation and the empirical standard deviation may reflect measurement error, which should systematically raise the standard deviation of the empirical data. In addition, most of the empirical consumption series include durables, which should raise the variability of consumption growth (Mankiw, 1982). By contrast, the U.S. consumption data omit durables, and for the United States we calculate $\sigma_{C}=0.0054$, closely matching our theoretical value.

Next, we turn to the first-order autocorrelation of consumption growth, applying again Theorem 3:

$$
\begin{aligned}
\rho_{C} & =\operatorname{corr}\left(\ln \frac{C_{t}}{C_{t-1}}, \ln \frac{C_{t-2}}{C_{t-1}}\right) \\
& =\left(\sigma_{C}^{2}\right)^{-1}\left(n^{2} \sigma_{N}^{2} \Gamma(0,1)+\Theta^{2} \sigma^{2} \iint_{D, D^{\prime} \in[0, \bar{D}]} \Gamma\left(D, D^{\prime}, 1\right) \frac{d D d D^{\prime}}{\bar{D}^{2}}\right) .
\end{aligned}
$$

Using our calibration choices, our model implies $\rho_{C}=0.34 . .^{25}$ This theoretical prediction lies well above the empirical estimate of -0.11 , found by averaging across the country-by-country autocorrelations in the Campbell dataset. Here too, both measurement error and the inclusion of durables are likely to bias the empirical correlations down. Again, the U.S. data, which omits durables, come much closer to matching our theoretical prediction. In the U.S. data, $\rho_{C}=0.22$.

23. We thank John Campbell for sharing this dataset with us.

24. We use quarterly data from the Campbell dataset. The quarterly data begins in 1947 for the United States, and begins close to 1970 for most of the other countries. The dataset ends in 1996.

25. The respective effects are $n^{2} \sigma_{N}^{2} \Gamma(0,1)=0.077 \times 10^{-4}$ and $\Theta^{2} \sigma^{2} / \int_{D, D^{\prime} \in[0, \tilde{D}]} \Gamma\left(D, D^{\prime}, 1\right)$ $d D d D^{\prime} / \bar{D}^{2}=0.048 \times 10^{-4}$. 
We turn now to the covariation between aggregate consumption growth and equity returns, $\operatorname{cov}\left(\ln \left[C_{t} / C_{t-1}\right], \ln R_{t}\right)$. We find

$\operatorname{cov}\left(\ln \frac{C_{t}}{C_{t-1}}, \ln R_{t}\right)=\Theta \sigma^{2} \int_{D \in[0, \bar{D}]} V(D, 0,1) \frac{d D}{\bar{D}}=0.13 \times 10^{-4}$,

assuming that in the short run the consumption growth of nonstockholders is uncorrelated with that of stockholders. The covariance estimate of $0.13 \times 10^{-4}$ almost matches the average covariance in the Campbell dataset, $0.14 \times 10^{-4}$. This time, however, the U.S. data do not "outperform" the rest of the countries in the Campbell dataset. For the United States, the covariance is $0.60 \times 10^{-4}$. However, all of these covariances come much closer to matching our model than to matching the benchmark model with instantaneous adjustment and measurement. The benchmark model with no delayed adjustment predicts that the quarterly covariance will be $\theta \sigma^{2} \simeq 50 \times 10^{-4}$.

What would an econometrician familiar with the consumption-CAPM literature conclude if he observed quarterly data from our $6 D$ economy, but thought he were observing data from the benchmark economy? First, he might calculate

$$
\hat{\gamma}=\frac{\pi}{\operatorname{cov}\left(\ln \left[C_{t} / C_{t-1}\right], \ln R_{t}\right)} \simeq 1000,
$$

and conclude that the coefficient of relative risk aversion is over 1000 . If he were familiar with the work of Mankiw and Zeldes (1991), he might restrict his analysis to stockholders and calculate

$$
\hat{\gamma}=\frac{\pi}{\operatorname{cov}\left(\ln \left[S_{t} / S_{t-1}\right], \ln R_{t}\right)} \simeq 300
$$

Finally, if he read Mankiw and Zeldes carefully, he would realize that he should also do a continuous-time adjustment (of the type suggested by Grossman, Melino, and Shiller, 1987), leading to another halving of his estimate. But, after all of this hard work, he would still end up with a biased coefficient of relative risk aversion: $300 / 2=150$. For this economy, the true coefficient of relative risk aversion is 3 !

These observations suggest that the literature on the equity-premium puzzle should be reappraised. Once one takes account of delayed adjust- 
ment, high estimates of $\gamma$ no longer seem anomalous. If workers in midlife take decades to respond to innovations in their retirement accounts, we should expect naive estimates of $\gamma$ that are far too high.

Defenders of the Euler-equation approach might argue that economists can go ahead estimating the value of $\gamma$ and simply correct those estimates for the biases introduced by delayed adjustment. However, we do not view this as a fruitful approach, since the adjustment delays are difficult to observe or calibrate.

For an active stock trader, knowledge of personal financial wealth may be updated daily, and consumption may adjust equally quickly. By contrast, for the typical employee who invests in a 401(k) plan, retirement wealth may be in its own mental account, ${ }^{26}$ and hence may not be integrated into current consumption decisions. This generates lags of decades or more between stock price changes and consumption responses. Without precise knowledge of the distribution of $D$-values, econometricians will be hard pressed to measure $\gamma$ accurately using the Eulerequation approach.

In summary, our model tells us that high imputed $\gamma$-values are not anomalous and that high-frequency properties of the aggregate data can be explained by a model with delayed adjustment. Hence, the equity premium may not be a puzzle.

Finally, we wish to note that our delayed-adjustment model is complementary to the theoretical work of other authors who have analyzed the equity-premium puzzle. ${ }^{27}$ Our qualitative approach has some similarity with the habit-formation approach (e.g., Constantinides, 1990; Abel, 1990; Campbell and Cochrane, 1999). Habit-formation models imply that slow adjustment is optimal because households prefer to smooth the growth rate (not the level) of consumption. In our $6 D$ model, slow adjustment is optimal only because decision costs make high-frequency adjustment too expensive.

\section{Review of Related Empirical Evidence}

In this section, we review two types of evidence that lend support to our model. In the first subsection we review survey evidence which suggests that investors know relatively little about high-frequency variation in their equity wealth. In the second subsection we show that equity innovations predict future consumption growth.

26. See Thaler (1992).

27. For other proposed solutions to the equity-premium puzzle see Kocherlakota (1996), Bernartzi and Thaler (1995), and Barberis, Huang and Santos (2000). 


\subsection{KNOWLEDGE OF EQUITY PRICES}

Consumers can't respond to high-frequency innovations in equity values if they don't keep close tabs on the values of their equity portfolios. In this subsection, we discuss survey evidence that suggests that consumers may know little about high-frequency variation in the value of their equity wealth. ${ }^{28}$ We also discuss related evidence that suggests that consumers may not adjust consumption in response to business-cyclefrequency variation in their equity holdings. All of this evidence is merely suggestive, since survey responses may be unreliable.

The 1998 Survey of Consumer Finances (SCF) was conducted during the last six months of 1998, a period of substantial variation in equity prices. In July the average value of the Wilshire 5000 equity index was 10,770 . The index dropped to an average value of 9,270 in September, before rising back to an average value of 10,840 in December. Kennickell, Starr-McCluer, and Surette (2000) analyze the 1998 SCF data to see whether self-reported equity wealth covaries with movements in stockmarket indexes. They find that the SCF equity measures are uncorrelated with the value of the Wilshire index on the respondents' respective interview dates. Only respondents who were active stock traders ( $\geq 12$ trades/year) showed a significant correlation between equity holdings and the value of the Wilshire index.

Dynan and Maki (2000) report related results. They analyze the responses to the Consumer Expenditure Survey (CEX) from the first quarter of 1996 to the first quarter of 1999. During this period, the U.S. equity markets rose over $15 \%$ during almost every 12 -month period. Nevertheless, when respondents were surveyed for the CEX, one-third of stockholders reported no change in the value of their securities during the 12month period before their respective interviews. ${ }^{29}$

Starr-McCluer (2000) analyzes data from the Michigan Survey Research Center (SRC) collected in the summer of 1997. One of the survey questions asked, "Have you [Has your family] changed the amount you spend or save as a result of the trend in stock prices during the past few years?" Among all stockholder respondents, 85.0\% said "no effect." Among stockholder respondents with most of their stock outside retirement accounts, $83.3 \%$ said "no effect." Even among stockholders with large portfolios ( $\geq \$ 250,000), 78.4 \%$ said "no effect."

28. We are grateful to Karen Dynan for pointing out much of this evidence to us.

29. For the purposes of this survey a change in the value of equity securities includes changes due to price appreciation, sales, and/or purchases. 


\subsection{THE EFFECT OF LAGGED EQUITY RETURNS ON CONSUMPTION GROWTH}

Dynan and Maki (2000) analyze household-level data on consumption growth from the CEX, and ask whether lagged stock returns affect future consumption growth. They break their results down for nonstockholders and stockholders. For stockholders with at least $\$ 10,000$ in securities a $1 \%$ innovation in the value of equity holdings generates a $1.03 \%$ increase in consumption of nondurables and services. However, this increase in consumption occurs with a lag. One third of the increase occurs during the first 9 months after the equity price innovation. Another third occurs 10 to 18 months after the innovation. Another quarter of the increase occurs 19 to 27 months after the innovation, and the rest of the increase occurs 28 to 36 months after the innovation.

We now turn to evidence from aggregate data. We look for a relationship between equity returns and future consumption growth. Specifically, we evaluate $\operatorname{Cov}\left(\ln \left[C_{t+h} / C_{t}\right], \ln R_{t+1}\right)$ for $h=1,2, \ldots, 25$.

Under the null hypothesis of $D=0$, the quarterly covariance between equity returns and consumption growth is predicted to be

$$
\begin{aligned}
\operatorname{Cov}\left(\ln \frac{C_{t+1}}{C_{t}}, \ln R_{t+1}\right) & =\frac{\Theta \sigma^{2}}{2} \\
& =\frac{(0.22)(0.16 / \sqrt{4})^{2}}{2} \\
& =0.0007 .
\end{aligned}
$$

The effects of time-aggregation bias are incorporated into this prediction. An equity innovation during period $t+1$ only affects consumption after the occurrence of the equity innovation. So the predicted covariance, $\operatorname{Cov}\left(\ln \left[C_{t+1} / C_{t}\right], \ln R_{t+1}\right)$, is half as great as it would be if consumption growth were measured instantaneously.

This time-aggregation bias vanishes once we extend the consumption growth horizon to two or more periods. So, if $D=0$ and $h \geq 2$,

$$
\begin{aligned}
\operatorname{Cov}\left(\ln \left[C_{t+h} / C_{t}\right], \ln R_{t+1}\right) & =\Theta \sigma^{2} \\
& =(0.22)(0.16 / \sqrt{4})^{2} \\
& =0.0014 .
\end{aligned}
$$

Hence the assumption $D=0$ implies that the profile of $\operatorname{Cov}\left(\ln \left[C_{t+h} / C_{t}\right], \ln R_{t+1}\right)$ for $h \geq 2$ should be flat. 
FIGURE 7 COVARIANCE OF $R_{t+1}$ AND $\ln \left(C_{t+h} / C_{t}\right)$

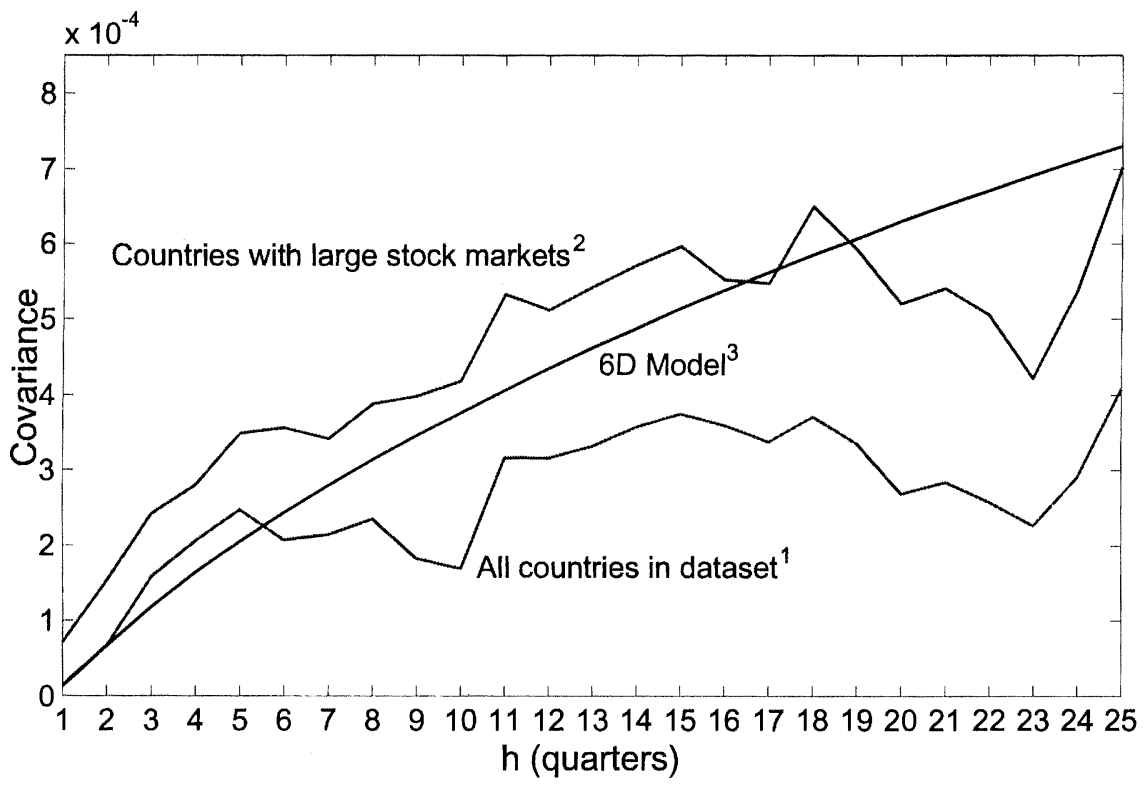

Notes:

1. Dataset is from Campbell (1999). Full dataset includes Australia, Canada, France, Germany, Italy, Japan, the Netherlands, Spain, Sweden, Switzerland, the United Kingdom, and the United States.

2. To identify countries with large stock markets, we ordered the countries by the ratio of stock-market capitalization to GDP (1993). The top half of the countries were included in our large-stock-market subsample: Switzerland (0.87), the United Kingdom (0.80), the United States $(0.72)$, the Netherlands (0.46), Australia (0.42), and Japan (0.40).

3. We assume that households have $D$-values that are uniformly distributed from 0 to 30 years.

Figure 7 plots the empirical values of $\operatorname{Cov}\left(\ln \left[C_{t+h} / C_{t}\right], \ln R_{t+1}\right)$ for $h \in$ $\{1,2, \ldots, 25\} .{ }^{30}$ We use the cross-country panel dataset created by Campbell (1999). ${ }^{31}$ Figure 7 plots the value of $\operatorname{Cov}\left(\ln \left[C_{t+h} / C_{t}\right], \ln R_{t+1}\right)$, averaging across all of the countries in Campbell's dataset: Australia, Canada, France, Germany, Italy, Japan, the Netherlands, Spain, Sweden, Switzerland, the United Kingdom, and the United States. ${ }^{32}$ Figure 7 also plots the

30. See Hall (1978) for early evidence that lagged stock returns predict future consumption growth. See Lettau and Ludvigson (2001) for a VAR approach that implies that lagged stock returns do not predict future consumption growth. Future work should attempt to reconcile our results with those of Lettau and Ludvigson.

31. We thank John Campbell for giving this dataset to us.

32. Specifically, we calculate $\operatorname{Cov}\left(\ln R_{t+1}, \ln \left[C_{t+h} / C_{t}\right]\right)$ for each country and each $h$-quarter horizon, $h \in\{1,2, \ldots, 25\}$. We then average across all of the countries in the sample. We use quarterly data from the Campbell dataset. The quarterly data begin in 1947 for the United States, and begin close to 1970 for most of the other countries. The dataset ends in 1996. 
average value of $\operatorname{Cov}\left(\ln \left[C_{t+h} / C_{t}\right], \ln R_{t+1}\right)$, averaging across all of the countries with large stock markets. Specifically, we ordered the countries in the Campbell dataset by the ratio of stock-market capitalization to GDP in 1993. The top half of the countries were included in our large-stock-market subsample: Switzerland (0.87), United Kingdom (0.80) United States (0.72), Netherlands (0.46), Australia (0.42), and Japan (0.40).

Two properties of the empirical covariances stand out. First, they slowly rise as the consumption growth horizon $h$ increases. Contrast this increase with the counterfactual prediction for the $D=0$ case that the covariance should plateau at $h=2$. Second, the empirical covariances are much lower than the covariance predicted by the $D=0$ case. For example, at a horizon of 4 quarters, the average empirical covariance is roughly 0.0002 , far smaller than the theoretical prediction of 0.0014 .

Figure 7 also plots the predicted ${ }^{33}$ covariance profile implied by the $6 D$ model. ${ }^{34}$ To generate this prediction we assume that $D$-values are uniformly distributed from 0 years to 30 years, as discussed in the previous section.

The $6 D$ model predicts that the covariance $\operatorname{Cov}\left(\ln \left[C_{t+h} / C_{t}\right], \ln R_{t+1}\right)$ slowly rises with the horizon $h$. To understand this effect, recall that the $6 D$ economy slowly adjusts to innovations in the value of equity holdings. Some consumers respond quickly to equity innovations, either because these consumers have low $D$-values, or because they have a

33. Corollary 7 gives

$$
\operatorname{Cov}\left(\ln \left[C_{t+h} / C_{t}\right], \ln R_{t+1}\right)=\Theta \sigma^{2} \int_{D \in[0, \bar{D}]}[e(1+D)-e(1)-e(1-h+D)+e(1-h)] \frac{d D}{D \bar{D}} .
$$

34. The following approximation for the covariances provides intuition for the orders of magnitude. In normalized units,

$$
\frac{1}{\Theta \sigma^{2}} \operatorname{cov}\left(\ln \frac{C_{t+h}}{C_{t}}, R_{t+1} \mid D\right) \simeq \max \left(\frac{h}{D}, 1\right) .
$$

When the $D^{\prime}$ s are uniformly distributed in $[0, \bar{D}]$,

$$
\begin{aligned}
\frac{1}{\Theta \sigma^{2}} \operatorname{cov}\left(\ln \frac{C_{t+h}}{C_{t}}, R_{t+1}\right) & =\int_{0}^{\bar{D}} \operatorname{cov}\left(\ln \frac{C_{t+h}}{C_{t}}, R_{t+1} \mid D\right) \frac{d D}{\bar{D}} \\
& \simeq \int_{0}^{\bar{D}} \max \left(\frac{h}{D}, 1\right) \frac{d D}{\bar{D}} \\
& =\frac{h}{\bar{D}}\left(1+\ln \frac{\bar{D}}{h}\right) .
\end{aligned}
$$

This approximation turns out to be quite good for $h \geq 2$. 
high $D$-value and are coincidentally coming up to a reset period. Other consumers respond with substantial lags. For our illustrative example, the full response will take 30 years. For low $h$, the $6 D$ model predicts that the covariance profile will be close to zero. As $h$ goes to infinity, the covariance profile asymptotes to the prediction of the instantaneous adjustment model, so $\lim _{h \rightarrow \infty} \operatorname{Cov}\left(\ln \left[C_{t+h} / C_{t}\right], \ln R_{t+1}\right)=\Theta \sigma^{2}=0.0014$. Figure 7 shows that our illustrative calibration of the $6 D$ model does a fairly good job of matching the empirical covariances.

This analysis has shown that the empirical data are completely inconsistent with the standard assumption of instantaneous adjustment. Lagged equity returns affect consumption growth at very long horizons: $\operatorname{Cov}\left(\ln \left[C_{t+h} / C_{t}\right], \ln R_{t+1}\right)$, rises slowly with $h$, instead of quickly plateauing at $h=2$. This slow rise is a key test of the $6 D$ framework.

We conclude from Figure 7 that the $6 D$ model successfully predicts the profile of $\operatorname{Cov}\left(\ln \left[C_{t+h} / C_{t}\right], \ln R_{t+1}\right)$ for $h=1,2, \ldots, 25$. However, the $6 D$ model fails to predict the profile of a closely related quantity, the normalized Euler covariance,

$\frac{1}{h} \operatorname{Cov}\left(\ln \frac{C_{t+h}}{C_{t}}, \sum_{i=1}^{h} \ln R_{t+i}\right)$

This $h$-period covariance generalizes the one-period Euler covariance, $\operatorname{Cov}\left(\ln \left[C_{t+1} / C_{t}\right], \ln R_{t+1}\right){ }^{35,36}$

The standard model with $D=0$ predicts that the $h$-period normalized Euler covariance will equal [(2h-1)/2h] $\Theta \sigma^{2}$ for all (integer) values of $h$. The factor $(2 h-1) / 2 h$ captures time-aggregation bias, which becomes proportionately less important as the horizon increases. By contrast, the $6 D$ model predicts that, if the $D^{\prime}$ s are uniformly distributed between 0 and $\bar{D}$ (e.g., $D=30$ years $=120$ quarters), the $h$-period normalized Euler covari-

35. We thank Monika Piazzesi, whose insightful discussion of this paper at the NBER Macroeconomics Annual Conference led us to add analysis of the covariance Euler equation to this final draft.

36. The Euler covariances link the equity premium to the coefficient of relative risk aversion. Consider the $h$-period Euler equation for a discrete-time model with instantaneous adjustment, $E_{t-1}\left[\hat{\beta}\left(C_{t+h} / C_{t}\right)^{-\hat{\gamma}} \exp \left(\sum_{i=1}^{h} \ln R_{t+i}^{a}\right)\right]=1$ (for all assets $a$ ). Manipulation of this equation implies

$$
\hat{\gamma}=\frac{\pi}{\operatorname{cov}\left(\left[\sum_{i=1}^{h} \ln R_{t+i}\right], \ln \left[C_{t+h} / C_{t}\right]\right) / h},
$$

where $\pi$ is the 1-period equity premium. 
ance should approximately ${ }^{37}$ equal $(h / 4 \bar{D})[3-2 \ln (h / \bar{D})] \Theta \sigma^{2}$ for $h<\bar{D}$. For both the standard model $(D=0)$ and the $6 D$ model, the normalized Euler covariance should rise monotonically with $h$, but this rise should be much steeper for the standard model.

The empirical data match neither prediction. In the twelve-country Campbell data, an initial rise in the Euler covariance from $h=1$ to $h=7$ is subsequently reversed for larger values of $h$. For $h>20$, the Euler covariances are very small in magnitude, with some negative point estimates. ${ }^{38}$

This result seems to contradict the encouraging results plotted in Figure 7. To understand this tension, we assume stationarity and decompose the $h$-period Euler covariance:

$$
\begin{aligned}
\operatorname{Cov}\left(\ln \frac{C_{t+h}}{C_{t}}, \sum_{i=1}^{h} \ln R_{t+i}\right) & =\sum_{i=1}^{h} \operatorname{Cov}\left(\ln \frac{C_{t+i}}{C_{t}}, \ln R_{t+1}\right) \\
& +\sum_{i=1}^{h-1} \operatorname{Cov}\left(\ln \frac{C_{t}}{C_{t-i}}, \ln R_{t+1}\right) .
\end{aligned}
$$

The $h$-period Euler covariance (i.e., the left-hand side) is zero for large $h$ 's, and the first sum on the right-hand side is positive (this is the quantity plotted in Figure 7). It follows that the second term on the righthand side should be negative:

37. We use the approximation above,

$$
\frac{1}{\Theta \sigma^{2}} \operatorname{cov}\left(\ln \frac{C_{t+h}}{C_{t}}, \ln R_{t+1}\right) \simeq \frac{h}{\bar{D}}\left(1+\ln \frac{\bar{D}}{h}\right),
$$

to get

$$
\begin{aligned}
\frac{1}{h \Theta \sigma^{2}} \operatorname{cov}\left(\ln \frac{C_{t+h}}{C_{t}}, \ln R_{t+1}+\ldots+\ln R_{t+h}\right) & =\frac{1}{h \Theta \sigma^{2}} \sum_{h^{\prime}=1}^{h} \operatorname{cov}\left(\ln \frac{C_{t+h^{\prime}}}{C_{t}}, \ln R_{t+1}\right) \\
& \simeq \frac{1}{h} \int_{0}^{h} \frac{h^{\prime}}{\bar{D}}\left(1+\ln \frac{\bar{D}}{h^{\prime}}\right) d h^{\prime} \\
& =\frac{h}{4 \bar{D}}\left(3-2 \ln \frac{h}{\bar{D}}\right) \text { for } h \leq \bar{D} \\
& =1-\frac{\bar{D}}{4 h} \text { for } h>\bar{D} .
\end{aligned}
$$

38. See Cochrane and Hansen (1992) for an early empirical analysis of the multiperiod Euler equation. Daniel and Marshall $(1997,1999)$ report that consumption Euler equations for aggregate data are not satisfied at the quarterly frequency but improve at the two-year frequency. Our results are consistent with theirs, but we find that this relatively good performance deteriorates as the horizon is lengthened. 
$\sum_{i=1}^{h-1} \operatorname{Cov}\left(\ln \frac{C_{t}}{C_{t-i}}, \ln R_{t+1}\right)<0$,

which can be verified in our sample. ${ }^{39}$ In words, lagged consumption growth negatively predicts the current stock return. Such predictability explains why the Euler covariance does not follow the profile predicted by the $6 \mathrm{D}$ model. Of course, this predictability is inconsistent with any model in which the stock market follows a martingale. Alternative frameworks, like Campbell and Cochrane's (1999) model of habit formation, Barberis, Huang, and Santos's (2001) prospect-theory model of asset pricing, or animal-spirits models, are needed to explain why lagged consumption growth negatively forecasts future stock returns.

\section{Conclusion}

Grossman and Laroque (1990) argue that adjustment costs might explain the equity-premium puzzle. Lynch (1996) and Marshall and Parekh (1999) have successfully numerically simulated discrete-time delayed adjustments models which confirm Grossman and Laroque's conjecture. We have described a continuous-time generalization of Lynch's (1996) model. We derive a complete analytic characterization of the model's dynamic properties. In addition, our continuous-time framework generates effects that are up to six times larger than those in discrete-time models.

We analyze an economy composed of consumers who update their consumption every $D$ periods. Using data from our economy, an econometrician estimating the coefficient of relative risk aversion (CRRA) from the consumption Euler equation would generate a multiplicative CRRA bias of $6 D$. Once we take account of this $6 D$ bias, the Euler equation tests are unable to reject the standard consumption model.

We have derived closed-form expressions for the first and second moments of this delayed-adjustment economy. The model matches most of the empirical moments of aggregate consumption and equity returns, including a new test which confirms the $6 D$ prediction that the covariance

39. For quarterly horizons $h \in\{5,10,15,20,25\}$, the average value of

$$
\sum_{i=1}^{h-1} \operatorname{Cov}\left(\ln \frac{C_{t}}{C_{t-i}}, \ln R_{t+1}\right)
$$

is $\{-0.9,-2.0,-4.6,-2.8,-3.6\} \times 10^{-4}$ for all of the countries in the Campbell dataset, and $\{-1.2,-2.4,-5.0,-3.0,-3.2\} \times 10^{-4}$ for the countries with large stock markets. 
between $\ln \left(C_{t+h} / C_{t}\right]$, and $R_{t+1}$ should slowly rise with $h$. The $6 D$ model fails long-horizon Euler-equation tests, but this failure is due to the interesting empirical regularity that high lagged consumption growth predicts low future equity returns.

Future work should test the new empirical implications of our framework, including the rich covariance lag structure that we have derived. Most importantly, our model implies that standard Euler-equation tests should be viewed very skeptically. Even small positive values of $D$ (e.g., $D=4$ quarters) dramatically bias the inferences that economists draw from Euler equations and the related Hansen-Jagannathan bounds.

\section{Appendix A. Proofs}

We use approximation to get analytic results. Let $\varepsilon \equiv \max (r, \rho, \theta \pi$, $\left.\sigma^{2}, \sigma^{2} \theta^{2}, \alpha\right)$. For annual data $\varepsilon \simeq 0.05$. We shall use the notation $f(\varepsilon)=$ $O_{<t}\left(\varepsilon^{k}\right)$, for $k \in \mathbb{R}$, to mean that $f$ is measurable with respect to the information known at time $t$, and there is $\varepsilon_{0} \geq 0$ and a constant $A>0$ such that for $\varepsilon \leq \varepsilon_{0} A$, we have $E_{0}\left[f^{2}\right]^{1 / 2} \leq A\left|\varepsilon^{k}\right|$. More concisely, the norms are in the $L_{2}$ sense. For instance:

$$
\begin{aligned}
e^{r t+\sigma z(t)} & =1+\sigma z(t)+r t+\frac{\sigma^{2} z(t)^{2}}{2}+O_{<s}\left(\varepsilon^{3 / 2}\right) \\
& =1+\sigma z(t)+O_{<s}(\varepsilon)=1+O_{<s}\left(\varepsilon^{1 / 2}\right) .
\end{aligned}
$$

We will often replace $O_{<t}\left(\varepsilon^{k}\right)$ by $O\left(\varepsilon^{k}\right)$ when there is a clear smallest time $t$ such that $f$ is measurable with respect to the information known at time $t$. For instance, we would write $e^{r t+\sigma z(t)}=1+\sigma z(t)+O(\varepsilon)$ to mean $e^{r t+\sigma z(t)}=$ $1+\sigma z(t)+O_{<t}(\varepsilon)$.

Also, we shall often use the function

$a(i) \equiv(1-|i|)^{+}$.

Finally, for $z$ a generic standard Brownian motion, we define $z_{[i, j]}=z(j)$ $-z(i)$, and remark that

$$
\operatorname{cov}\left(z_{[i-D, i]}, z_{\left[j-D^{\prime}, j\right]}\right)=\min \left(\left(D-(i-j)^{+}\right)^{+},\left(D^{\prime}-(j-i)^{+}\right)^{+}\right),
$$

as both are equal to the measure $[i-D, i] \cap\left[j-D^{\prime}, j\right]$.

\section{A.1 PROOF OF PROPOSITION 1}

Denote by $v(w)=E \int_{0}^{\infty}\left[e^{-\rho t} c_{t}^{1-\gamma} /(1-\gamma)\right] d t$ the expected value of the utils from consumption under the optimal policy, assuming the first reset 
date is $t=0$. So $v(\cdot)$ is the value function that applies at reset dates. Say that the agent puts $S$ in the checking account, and the rest, $w-S$, in the mutual fund. Call $M$ the (stochastic) value of the mutual fund at time $D$. By homotheticity, we have $v(w)=v \cdot w^{1-\gamma} /(1-\gamma)$. We have

$v(w)=\int_{0}^{D} e^{-\rho t} \frac{c_{t}^{1-\gamma}}{1-\gamma} d t+e^{-\rho D} E\left[v\left(w^{\prime}\right)\right]$

with

$w^{\prime}=M+S e^{r D}-\int_{0}^{D} c_{t} e^{r(D-t)} d t$

Optimizing over $c_{t}$ for $t \in[0, D)$, we get $c_{t}^{-\gamma}=E\left[v^{\prime}\left(w^{\prime}\right)\right] e^{(r-\rho)(D-t)}$, so that consumption growth is that of the Ramsey model: $c_{t}=\alpha w e^{[(r-\rho) / \gamma] t}$ for some $\alpha$ (by the implicit-function theorem one can show that it is a continuous function of $D$, and it has Merton's value when $D=0$ ). To avoid bankruptcy, we need $S \geq S_{0}=\int_{0}^{D} c_{t} e^{-r t} d t$. Imagine that the consumer starts by putting aside the amount $S_{0}$. Then, he has to manage optimally the remaining amount, $w-S_{0}$. Given some strategy, he will end up with a stochastic wealth $w^{\prime}$, and he has to solve the problem of maximizing $v E\left[w^{\prime 1-\gamma} /(1-\gamma)\right]$. But this is a finite-horizon Merton problem with utility derived from terminal wealth, whose solution is well known: the whole amount $w-S_{0}$ should be put in a mutual fund with constant rebalancing, with a proportion of stocks $\theta=\pi /\left(\gamma \sigma^{2}\right)$. In particular, only the amount $S_{0}$ is put in the checking account.

\section{A.2 PROOF OF PROPOSITION 9}

The basis of our calculations is the representation formula for consumption, Proposition 9. To prove it we shall need the following

LEMMA 10 We have

$w_{i t+s}=w_{i t}\{1+\theta \sigma[z(t+s)-z(t)]+O(\varepsilon)\}$.

PROOF If the agent doesn't check her portfolio between $t$ and $t+s$, we have

$$
\begin{aligned}
w_{i t+s} & =w_{i t} e^{\left(r+\theta \pi-\theta^{2} \sigma^{2} / 2\right) s+\sigma \theta[z(t+s)-z(t)]} \\
& =w_{i t}\{1+\sigma \theta[z(t+s)-z(t)]+O(\varepsilon)\} .
\end{aligned}
$$


When the agent checks her portfolio at time $\tau$, she puts a fraction $f=$ $\int_{0}^{D} \alpha e^{-r t+[(r-\rho) / \gamma] t} d t=O(\varepsilon)$ in the checking account, so that

$$
\begin{aligned}
w_{i \tau^{+}} & =w_{i \tau^{-}}(1-f) \\
& =w_{i \tau^{-}}[1+O(\varepsilon)] .
\end{aligned}
$$

Pasting together (37) and (39) at different time intervals, we see that (37) holds between two arbitrary dates (i.e., possibly including reset dates) $t$ and $t+s$, and the lemma is proven.

We can now proceed to the

PROOF OF PROPOSITION 9 Say that $i \in[0, D]$ has her latest reset point before $t-1$ at $t_{i}=t-1-i$. The following reset points are $t_{i}+m D$ for $m$ $\geq 1$, and for $s \geq t-1$ we have [the first $O(\varepsilon)$ term capturing the deterministic increase of consumption between reset dates]

$$
\begin{aligned}
\frac{c_{i s}}{\alpha} & =\left(w_{i t_{i}}+\sum_{m \geq 1}\left(w_{i t_{i}+m D}-w_{i t_{i}+(m-1) D}\right) 1_{s \geq t_{i}+m D}\right)[1+O(\varepsilon)] \\
& =w_{i t_{i}}+\sum_{m \geq 1} w_{i t_{i}}\left[\theta \sigma z_{\left[t_{i}+(m-1) D, t_{i}+m D\right]}+O(\varepsilon)\right] 1_{s \geq t_{i}+m D}+O(\varepsilon),
\end{aligned}
$$

so that, using the notation $\zeta_{i m} \equiv w_{i t_{i}} \theta \sigma z_{\left[t_{i}+(m-1) D, t_{i}+m D\right]}$,

$$
\begin{aligned}
\int_{t_{i}}^{T} \frac{c_{i s}}{\alpha} d s+O(\varepsilon) & =\left(T-t_{i}\right) w_{i t_{i}}+\sum_{m \geq 1} \zeta_{i m} \int_{t_{i}}^{T} 1_{s \geq t_{i}+m D} d s \\
& =\left(T-t_{i}\right) w_{i t_{i}}+\sum_{m \geq 1} \zeta_{i m}\left(T-\left(t_{i}+m D\right)\right)^{+},
\end{aligned}
$$

and we get 


$$
\begin{aligned}
c_{i t+1}-c_{i t}+O(\varepsilon) & =\left(\int_{t_{i}}^{t+1}-2 \int_{t_{i}}^{t}+\int_{t_{i}}^{t-1}\right) c_{i s} d s \\
& =\alpha \sum_{\substack{m \geq 1 \\
\tau_{i m}=t_{i}+m D}} \zeta_{i m}\left[\left(t+1-\tau_{i m}\right)^{+}-2\left(t-\tau_{i m}\right)^{+}+\left(t-1-\tau_{i m}\right)^{+}\right] \\
& =\alpha \sum_{m \geq 1} \zeta_{i m} a\left(t-\left(t_{i}+m D\right)\right), \\
& =\alpha \sum_{m \geq 1} \zeta_{i m} a(1+i-m D),
\end{aligned}
$$

because $t_{i}=t-1-i$.

Let $w_{t-D-1}=w_{i_{0}, t-D-1}$, which implies that $w_{i, t-D-1}=w_{t-D-1}[1+O(\varepsilon)]$ for all $i$. Note that $i_{0}$ is an arbitrarily selected index value. We now get the expression for consumption growth,

$$
\begin{aligned}
C_{t+1}-C_{t} & =\int_{0}^{D}\left(c_{i t+1}-c_{i t}\right) \frac{d i}{D} \\
& =\alpha \sum_{m \geq 1} \int_{0}^{D} w_{t-D-1} \theta \sigma z_{[t-1-i+(m-1) D, t-1-i+m D]} a(1+i-m D) \frac{d i}{D}+O(\varepsilon) .
\end{aligned}
$$

Defining $j \equiv D-1-\mathrm{i}$, and noting that the above expressions paste together, we have

$$
\frac{C_{t+1}-C_{t}}{\alpha w_{t-D-1}}=\int_{-1}^{1} \theta \sigma z_{[t+j-D, t+j]} a(j) \frac{d j}{D}+O(\varepsilon)
$$

One can likewise calculate

$$
\frac{C_{t}}{\alpha w_{t-D-1}}=1+O(\sqrt{\varepsilon})
$$


$\ln \frac{C_{t+1}}{C_{t}}=\int_{-1}^{1} \theta \sigma z_{[t+j-D, t+j]} a(j) \frac{d j}{D}+O(\varepsilon)$.

\section{A.3 PROOF OF THEOREM 2}

Use Proposition 9, $\ln R_{t+1}=\sigma z_{[t, t+1]}+O(\varepsilon)$, to get

$$
\begin{aligned}
\operatorname{cov}\left(\ln \frac{C_{t+1}}{C_{t}}, \ln R_{t+1}\right) & =\theta \sigma^{2} \int_{-1}^{1} a(i) \operatorname{cov}\left(z_{[t+i-D, t+i]}, z_{[t, t+1]}\right) \frac{d i}{D} \\
& +O_{<t}\left(\varepsilon^{3 / 2}\right)
\end{aligned}
$$

with

$$
\begin{aligned}
\int_{-1}^{1} a(i) \operatorname{cov}\left(z_{[t+i-D, t+i]}, z_{[t, t+1]}\right) \frac{d i}{D} & =\int_{0}^{1} a(i) \min (D, i) \frac{d i}{D} \quad \text { by }(34) \\
& =\frac{3(1-D)+D^{2}}{6} \quad \text { if } \quad D \leq 1 \\
& =\frac{1}{6 D} \quad \text { if } \quad D \geq 1
\end{aligned}
$$

Using (1) and (6), this leads to the expression (2).

\section{A.4 PROOF OF THEOREM 3}

First we need

LEMMA 11 We have, with d defined in (11), for $D \in \mathbb{R}$,

$$
\int_{\mathbb{R}} a(i) a(i+D) d i=d^{\prime \prime}(D) .
$$

PROOF OF LEMMA 11 Define, for $D \in \mathbb{R}$,

$$
g(D) \equiv \int_{\mathbb{R}} a(i) a(i+D) d i
$$

First, note that $g$ is even because $a$ is. In addition, for $D \geq 2, g(D)=0$ : for the integrand to be nonzero in (40), we need both $|i|<1$ and $|i+D|<$ 1 , which is impossible for $D \geq 2$. 
For a general $D$, we derive (in the sense of the theory of distributions, with Dirac's $\delta$-function $\left.{ }^{40}\right) g$ over $D$, starting from (40):

$$
\begin{aligned}
g^{(4)}(D) & =\int_{\mathbb{R}} a(i) a^{(4)}(i+D) d i \\
& =\int_{\mathbb{R}} a^{\prime \prime}(i) a^{\prime \prime}(i+D) d i \quad \text { by integration by parts } \\
& =\sum_{j=0}^{4}\left(\begin{array}{l}
4 \\
j
\end{array}\right)(-1)^{j} \delta(j-2+D)
\end{aligned}
$$

by direct calculation (or combinatorial insight) using $a^{\prime \prime}(x)=\delta(x+1)-$ $2 \delta(x)+\delta(x-1)$. We now integrate $g^{(4)}(D)$, which gives

$$
\begin{aligned}
g(D) & =\sum_{j=0}^{4}\left(\begin{array}{l}
4 \\
j
\end{array}\right) \frac{(-1)^{j}}{2 \times 3 !}|j-2+D|^{3}+\sum_{j=0}^{3} b_{j} D^{j} \\
& =d^{\prime \prime}(D)+\sum_{j=0}^{3} b_{j} D^{j}
\end{aligned}
$$

where the $b_{j}$ are integration constants. But the condition $g(D)=0$ for $D \geq$ 2 forces the $b_{j}^{\prime}$ s to be 0 , which concludes the proof.

The rest of the proof is in two steps. First we prove (41)-(42), then we calculate this expression of $p(D, t)$.

Step 1. Using (25) at $t$ and $t+h$, we get

$$
\operatorname{cov}\left(\ln \frac{C_{t+1}}{C_{t}}, \ln \frac{C_{t+1+h}}{C_{t+h}}\right)=\theta^{2} \sigma^{2} \Gamma(D, h)+O\left(\varepsilon^{3 / 2}\right)
$$

with 


$$
\begin{aligned}
\Gamma(D, h) & =\operatorname{cov}\left(\int_{-1}^{1} a(i) z_{[t+i-D, t+i]} \frac{d i}{D}, \int_{-1}^{1} a(j) z_{[t+h+j-D, t+h+j]} \frac{d j}{D}\right) \\
& =\int_{-1}^{1} \int_{-1}^{1} a(i) a(j) \operatorname{cov}\left(z_{[t+i-D, t+i]}, z_{[t+h+j-D, t+h+j]}\right) \frac{d i}{D} \frac{d j}{D}
\end{aligned}
$$

so using (34) we get

$$
\Gamma(D, h)=\frac{p(D, h)}{D^{2}}
$$

with

$$
p(D, h) \equiv \iint_{i, j \in[-1,1]} a(i) a(j)(D-|i-j-h|)^{+} d i d j .
$$

Step 2. Our next step is to calculate $p(D, h)$. Start with the case $D \geq h+2$ : then $(D-|i-j-h|)^{+}=D-|i-j-h|$, as $\left.|i-j-h| \leq 1+1+h \leq D\right)$, and given $\iint_{i, j \in[-1,1]} a(i) a(j) d i d j=\left(\int_{i \in[-1,1]} a(i) d i\right)\left(\int_{i \in[-1,1]} a(j) d j\right)=1$, we get

$p(D, h)=D-A(h) \quad$ for $\quad D \geq h+2$

with

$$
A(h)=\iint_{i, j \in \mathbb{R}}|i-j-h| a(i) a(j) d i d j .
$$

Going back to a general $D>0$, we get from (42)

$$
\begin{aligned}
p^{\prime \prime}(D) & =\iint_{i, j \in \mathbb{R}} a(i) a(j) \delta(D-|i-j-h|) d i d j \\
& =\int_{\mathbb{R}} a(i)[a(i+D-h)+a(i-D-h)] d i \\
& =\int_{\mathbb{R}} a(i)[a(i+D-h)+a(i+D+h)] d i
\end{aligned}
$$

because $a$ is even and by an application of change in variables. So from Lemma $11, p^{\prime \prime}(D)=d^{\prime \prime}(D-h)+d^{\prime \prime}(D+h)$, and 
$p(D, h)=d(D+h)+d(D-h)+d_{0}+d_{1} D$

for some real numbers $d_{0}, d_{1}$. Equation (43) gives us $d_{1}=0$, since $d^{\prime}(x)=\frac{1}{2}$ for $x>2$. Finally, $p(0)=0$ gives $A(h)=-d_{0}=d(h)+d(-h)$, which concludes the proof.

\section{A.5 PROOF OF COROLLARY 4}

$\Gamma(D, 0)$ is monotonic by direct calculation from the result in Theorem 3 . Theorem 3 also implies

$\Gamma(D, 0)=\frac{2}{3}-\frac{D^{2}}{6}+\frac{D^{3}}{20}$ for $\quad D \in[0,1]$.

Alternatively, this result can be obtained more directly from the calculation at the end of the proof of Theorem 3 .

\section{A.6 PROOF OF PROPOSITION 5}

Extend the argument used to prove Theorem 2. To calculate the correlation coefficient, use the variance results from Corollary 4.

\section{A.7 PROOF OF THEOREM 6}

Because $V\left(s_{1}, s_{2}\right)=V\left(s_{1}, 1\right)-V\left(s_{2}, 1\right)$, it is enough to fix $s_{2}=1$. We use the notation $s=s_{1}$. Recall (25), so that

$\operatorname{cov}\left(\ln \frac{C_{[t, t+1]}}{C_{[t-1, t]}}, \ln R_{[t+s, t+1]}\right)=\frac{\theta \sigma^{2}}{D} W(s)+O\left(\varepsilon^{3 / 2}\right)$

with

$$
\begin{aligned}
W(s) & =D \int_{-1}^{1} a(i) \operatorname{cov}\left(z_{[t+i-D, t+i]} z_{[t+s, t+1]}\right) \frac{d i}{D} \\
& =\int_{-1}^{1} a(i)(i-\max (i-D, s))^{+} d i .
\end{aligned}
$$

So, using the Heaviside function- $H(x) \equiv 1$ if $x \geq 0,0$ if $x<0$ (so that $H^{\prime}=\delta$ ) -

$$
\begin{aligned}
W^{\prime}(s) & =-\int a(i) H(i-\max (i-D, s)) H(s-i+D) d i \\
& =-\int a(i) H(i-s) H(s-i+D) d i
\end{aligned}
$$


and

$$
\begin{aligned}
W^{\prime \prime}(s) & =\int a(i)[\delta(i-s) H(s-i+D)-\delta(s-i+D) H(i-s)] d i \\
& =a(s)-a(s+D) .
\end{aligned}
$$

Introducing the function $e$ defined in (15), which satisfies $e^{\prime \prime}=a$, we get

$W(s)=e(s)-e(s+D)+W_{0}+W_{1} s$

for some constants $W_{0}, W_{1}$. Observe that for $s \geq 1$, (44) gives $W(s)=0$, so (45) gives us $W_{1}=0$ (and $W_{0}=D / 2$ ). This allows us to conclude the proposition.

\section{A.8 PROOF OF COROLLARY 7}

Immediate application of the preceding theorem.

\section{A.9 PROOF OF THEOREM 8}

The expression (23) is derived exactly as in Proposition 9. The only new work is to calculate $\Gamma\left(D, D^{\prime}, h\right)$. Using (34), we get

$\Gamma\left(D, D^{\prime}, h\right)=\frac{p\left(D, D^{\prime}, h\right)}{D D^{\prime}}$

with

$p\left(D, D^{\prime}, h\right)=\int_{i, j \in[-1,1]} a(i) a(j) \min \left(\left(D-(i-j-h)^{+}\right)^{+},\left(D^{\prime}-(j-i+h)^{+}\right)^{+} d i d j\right.$.

To calculate $p$, we derive (again, $H(x)=1_{x \geq 0}$ is Heaviside's function)

$p_{D^{\prime}}=\int a(i) a(j) H\left(\left(\left(D-(i-j-h)^{+}\right)^{+}-\left(D^{\prime}-(j-i+h)^{+}\right)^{+}\right)\right.$

$H\left(D^{\prime}-(j-i+h)^{+}\right) d i d j$

and 


$$
\begin{aligned}
p_{D^{\prime} D^{\prime}}= & \int_{\delta\left(D^{\prime}-(j-i+h)^{+}\right) d i d j} a(j) H\left(\left(D-(i-j-h)^{+}\right)^{+}-\left(D^{\prime}-(j-i+h)^{+}\right)^{+}\right) \\
& -\int a(i) a(j) \delta\left(\left(D-(i-j-h)^{+}\right)^{+}-\left(D^{\prime}-(j-i+h)^{+}\right)^{+}\right) \\
& H\left(D^{\prime}-(j-i+h)^{+}\right) d i d j \\
= & \int a(i)\left[a\left(i+D^{\prime}-h\right)-a\left(i+D^{\prime}-D-h\right)\right] d i .
\end{aligned}
$$

So Lemma 11 gives

$$
p=d\left(D^{\prime}-h\right)-d\left(D^{\prime}-D-h\right)+e_{0}+e_{1} D^{\prime}
$$

where $e_{0}, e_{1}$ are functions of $D$ and $h$. As $p=0$ for $D^{\prime}=0$, we get $e_{0}=$ $-d(-h)+d(-D-h)=-d(h)+d(D+h)$, as $d$ is even. As we should have $p(D, D, h)=p(D, h)$ for $p$ in (42), we can conclude $e_{1}=0$ and deduce the value of $e_{0}$, so Theorem 8 is proven.

\section{A.10 DERIVATION OF THE UTILITY LOSSES}

A fully rigorous derivation, e.g. of the type used by Rogers (2001), is possible here. Such a derivation begins with the Bellman equation (35), and then uses a Taylor expansion to derive an expression for $v$ of the type $v=v_{0}+v_{1} D+O\left(v^{2}\right)$. This approach is tedious and not very instructive about the economic origins of the losses, which is why we present the following more heuristic proof.

Equation (28) is standard (e.g., see Cochrane, 1989). For completeness's sake, though, let us mention a way to derive it. We want to calculate $U(C)-U\left(C^{\prime}\right)$, where $C=\left(c_{t}\right)_{t \geq 0}$ is the optimum vector of (stochastic) consumption flows, $U(C)=E\left[\int_{0}^{\infty} e^{-\rho t} u\left(c_{t}\right)\right]$, and $C^{\prime}$ is another vector that can be bought with the same Arrow-Debreu prices $p$. For $C$ and $C^{\prime}$ close, we have

$$
\begin{aligned}
\Delta U & \equiv U\left(C^{\prime}\right)-U(C) \\
& =U^{\prime}(C)\left(C^{\prime}-C\right)+\left(C^{\prime}-C\right)^{\prime} \cdot U^{\prime \prime}(C) \cdot \frac{C^{\prime}-C}{2}+O\left(\left(C^{\prime}-C\right)^{3}\right) .
\end{aligned}
$$

By optimality of $C$ we have $U^{\prime}(C)=\lambda p$ for some $p$, and $p C=p C^{\prime}=$ initial wealth $=W$; thus we have $U^{\prime}(C)\left(C-C^{\prime}\right)=0$. Expressing $U^{\prime \prime}$ finally gives 
$\Delta U=\frac{1}{2} E\left[\int_{0}^{\infty} e^{-\rho t} u^{\prime \prime}\left(c_{t}\right)\left(c_{t}-c_{t}^{\prime}\right)^{2} d t\right]$.

A change $\Delta W$ in the initial wealth creates, by homotheticity of the optimal policy, a change in consumption $\Delta c_{t} / c_{t}=\Delta W / W$, hence a change in utility

$\Delta U=E\left[\int_{0}^{\infty} e^{-\rho t} u^{\prime}\left(c_{t}\right) c_{t} \frac{\Delta W}{W} d t\right]$

So the suboptimality of plan $C^{\prime}$ is equivalent to a wealth loss [using $u^{\prime}(c)=c^{-\gamma}$ of

$$
\begin{aligned}
\Lambda_{c} & \equiv-\frac{\Delta W}{W}=-\frac{1}{2} \frac{E\left[\int_{0}^{\infty} e^{-\rho t} u^{\prime \prime}\left(c_{t}\right) c_{t}^{2}\left(\frac{c_{t}-c_{t}^{\prime}}{c_{t}}\right)^{2} d t\right]}{E\left[\int_{0}^{\infty} e^{-\rho t} u^{\prime}\left(c_{t}\right) c_{t} d t\right]} \\
& =\frac{\gamma}{2}\left\langle\left(\frac{c_{t}-c_{t}^{\prime}}{c_{t}}\right)^{2}\right\rangle
\end{aligned}
$$

where the weights in the mean $<\cdot>$ are given by $\left\langle X_{t}\right\rangle=E\left[\int_{0}^{\infty} e^{-\rho t} c_{t}^{1-\gamma} X_{t} d t\right] /$ $E\left[\int_{0}^{\infty} e^{-\rho t} c_{t}^{1-\gamma} d t\right]$. This proves equation (28).

We now derive $\left\langle\Delta c_{t}^{2} / c_{t}^{2}\right\rangle$, with $\Delta c_{t}=c_{t}^{\prime}-c_{t}$. With latest reset at time $\tau$,

$$
\begin{aligned}
\frac{\Delta c_{t}}{\alpha} & =\frac{c_{t}^{\prime}-c_{t}}{\alpha}=\left(w_{\tau}^{\prime}-w_{t}\right)[1+O(\varepsilon)] \\
& =\left(w_{\tau}-w_{t}+w_{\tau}^{\prime}-w_{\tau}\right)[1+O(\varepsilon)] .
\end{aligned}
$$

Now application of Lemma 10 gives (sparing the reader the tedious derivation),

$$
\begin{aligned}
\left\langle\frac{\left(w_{\tau}-w_{t}\right)^{2}}{w_{t}^{2}}\right\rangle & =E\left[\int_{0}^{D}\left(\int_{0}^{t} \theta \sigma d z_{s}\right)^{2} \frac{d t}{D}\right]+O\left(\varepsilon^{2}\right) \\
& =\frac{\theta^{2} \sigma^{2} D}{2}+O\left(\varepsilon^{2}\right) .
\end{aligned}
$$

Defining $\Psi$ such that $E\left[c_{t}^{1-\gamma}\right]=c_{0}^{1-\gamma} e^{(\rho-\psi) t}$, with $\Psi>0$, we get 


$$
\begin{aligned}
\left\langle\left(w_{\tau}^{\prime}-w_{\tau}\right)^{2} / w_{t}^{2}\right\rangle & =\left\langle\alpha^{2} \theta^{2} \sigma^{2} t D\right\rangle \\
& =\alpha^{2} \theta^{2} \sigma^{2} D \frac{\int_{0}^{\infty} e^{-\Psi t} t d t}{\int_{0}^{\infty} e^{-\Psi t} d t} \\
& =\alpha^{2} \theta^{2} \sigma^{2} D / \Psi \\
& =\theta^{2} \sigma^{2} D O(\varepsilon)=O\left(\varepsilon^{2}\right) .
\end{aligned}
$$

The cross term $\left\langle\left(w_{\tau}-w_{t}\right)\left(w_{\tau}^{\prime}-w_{\tau}\right)\right\rangle=0$.

So we have the important (and general in these kinds of problems) fact that the first-order contribution to the welfare loss is the direct impact of the delayed adjustment - the $w_{\tau}-w_{t}$ term-whereas the indirect impact (where a suboptimal choice of consumption creates modifications in future wealth) is second order. In other terms,

$$
\begin{aligned}
\left\langle\Delta c_{t}^{2} / c_{t}^{2}\right\rangle & =\left.\left\langle\Delta c_{t}^{2} / c_{t}^{2}\right\rangle\right|_{\text {without modification of the wealth process }}+O\left(\varepsilon^{2}\right) \\
& =\left\langle\left(w_{\tau}-w_{t}\right)^{2} / w_{t}^{2}\right\rangle+O\left(\varepsilon^{2}\right) \\
& =\theta^{2} \sigma^{2} D / 2+O\left(\varepsilon^{2}\right) .
\end{aligned}
$$

Using (28), we get (29).

\section{Appendix B. Model with Immediate Adjustment in Response to Large Changes in Equity Prices}

Suppose that people pay greater attention to "large" movements in the stock markets (because they are more salient, or because it is more rational to do so). How does our bias change? We propose the following tractable way to answer this question. Say that the returns in the stock market are

$d R_{t}=(\mu+r) d t+\sigma d z_{t}+d j_{t}$

where $j_{t}$ is a jump process with arrival rate $\lambda$. For instance, such jumps may correspond to crashes, or to "sharp corrections," though we need not have $E\left[d j_{t}\right]<0$. To be specific, when a crash arrives, the return falls by $J$ (to fix ideas, say $J=0.1-0.3$ ). To model high attention to crashes, we say that consumption adjusts to $d z_{t}$ shocks every $D$ periods, and adjusts to $d j$ shocks immediately ( $D=0$ for those Poisson events).

Denote by $\sigma_{\mathrm{B}}^{2}$ the variance of Brownian shocks, and by $\sigma_{\mathrm{I}}^{2}=E\left[d j_{t}^{2}\right] / d t$ $=\lambda J^{2}$ the variance of jump shocks. The total variance of the stock market 
is $\sigma^{2}=\sigma_{\text {tot }}^{2}=\sigma_{\mathrm{B}}^{2}+\sigma_{\mathrm{J}}^{2}$, assuming for simplicity that the two types of shocks are independent. The equity premium is $\pi=\mu-\lambda J$. By writing down the standard value function for the Merton problem, one sees that the optimal equity share, $\theta$, is now the solution of a nonlinear equation

$\pi-\gamma \sigma_{\mathrm{B}}^{2} \theta-\lambda J\left[(1-\theta J)^{-\gamma}-1\right]=0$.

For tractability, we use the approximation $J<<1$ (which is reasonable, since a typical value for $J$ is 0.1 to 0.25 ). We get the analogue of the simple formula (1):

$\theta=\frac{\pi}{\gamma \sigma_{\text {tot }}^{2}}$

plus higher-order terms in J. One can show that formula (22), which was derived in the case of assets with Brownian shocks, carries over to the case of a mix of Brownian shocks and jumps. Thus we get, to first order,

$\frac{\hat{\gamma}}{\gamma}=\left(\frac{\sigma_{\mathrm{B}}^{2}}{\sigma_{\text {tot }}^{2}} \frac{1}{b(D)}+\frac{\sigma_{\mathrm{I}}^{2}}{\sigma_{\text {tot }}^{2}} \frac{1}{b(0)}\right)^{-1}$

with $b(0)=2$ and $\sigma_{\text {tot }}^{2}=\sigma_{\mathrm{B}}^{2}+\sigma_{\mathrm{J}}^{2}$. Thus, the new bias is the harmonic mean of the $b(D)=6 D$ (if $D \geq 1$ ) bias for "normal" Brownian shocks, and the shorter $b(0)=2$ bias of the Brownian shocks.

As a numerical illustration, say a "jump" corresponds to a monthly change in the stock market of more than $J=25 \%$ in absolute value. This corresponds, empirically, to an estimate of $\lambda=0.53 \% /$ year ( 5 months since 1925), i.e. a crash every 14 years. Then $\sigma_{\mathrm{J}}^{2} / \sigma_{\text {tot }}^{2}=\lambda J^{2} / \sigma^{2}=0.014$. Take $D=4$ quarters as a baseline. The new $\hat{\gamma} / \gamma$ becomes 20.6 , which is close to the old ratio of 24 .

\section{Appendix C: Expression of the Bias in the Lynch Setup when $D \geq 1$}

In Lynch's (1996) discrete-time setup, agents consume every month and adjust their portfolio every $T$ months. The econometric observation period is time-aggregated periods of $F$ months, so $D=T / F$.

Say consumer $i \in\{1, \ldots, T\}$ adjusts her consumption at $i+n T, n \in$ $\mathbb{Z}$. Say the econometrician looks at period $\{1, \ldots, F\}$. The aggregate per capita consumption over this period is 
$C_{F}=\frac{1}{T} \sum_{i=1}^{T} \sum_{s=1}^{F} c_{i s}$

The returns are

$\ln R_{F}=\sum_{s=1}^{F} r_{s}$

where $r_{s}=\ln R_{s}$. Call $C_{i F}=\sum_{s=1}^{F} c_{i s}$ the consumption of agent $i$ in the period.

For $i>F, \operatorname{cov}\left(C_{i F}, \ln R_{F}\right)=0$, because agent $i$ did not adjust her consumption during the period.

For $1 \leq i \leq F$, we have $c_{i t}=1+O(\varepsilon)$ (normalizing) when $t<i$, and $c_{i t}=$ $1+\theta \sum_{s=1}^{i} r_{s}+O(\varepsilon)$ when $t \geq i$, where the $O(\varepsilon)$ terms incorporate the deterministic part of consumption growth. The stochastic part, in $r_{s}$, has the order of magnitude $\sigma=O\left(\varepsilon^{1 / 2}\right)$, and dominates those terms. Information about stock returns up to $i$ will affect only consumption from time $i$ to $F$, so, denoting by $\Delta C_{i F}$ the difference in total consumption between a given period of length $F$ and the previous one,

$$
\begin{aligned}
\operatorname{cov}\left(\Delta C_{i F}, \ln R_{F}\right) & =\operatorname{cov}\left((F+1-i) \theta \sum_{s=1}^{i} r_{s^{\prime}} \sum_{s=1}^{F} r_{s}\right) \\
& =\theta \sigma^{2} i(F+1-i) \quad \text { for } \quad 1 \leq i \leq F
\end{aligned}
$$

So

$$
\begin{aligned}
\operatorname{cov}\left(\Delta C_{F}, \ln R_{F}\right) & =\frac{1}{T} \sum_{i=1}^{T} \theta \sigma^{2} i(F+1-i) 1_{1 \leq i \leq F} \\
& =\frac{\theta \sigma^{2}}{T} \sum_{i=1}^{F}(F+1) i-i^{2} \\
& =\frac{\theta \sigma^{2}}{T}\left((F+1) \frac{F(F+1)}{2}-\frac{F(F+1)(2 F+1)}{6}\right) \\
& =\theta \sigma^{2} \frac{F(F+1)(F+2)}{6 T} .
\end{aligned}
$$


But given that the mean per-period consumption $c_{i t}=1+O\left(\varepsilon^{1 / 2}\right)$, the aggregate consumption is $C_{F}=F+O_{<0}\left(\varepsilon^{1 / 2}\right)$, and

$$
\begin{aligned}
\operatorname{cov}\left(\Delta C_{F} / C_{F}, \ln R_{F}\right) & \simeq \operatorname{cov}\left(\Delta C_{F}, \ln R_{F}\right) / F \\
& =\theta \sigma^{2} \frac{(F+1)(F+2)}{6 T} .
\end{aligned}
$$

The naive econometrician would predict $\operatorname{cov}\left(\Delta C_{F} / C_{F}, \ln R_{F}\right)=\theta \sigma^{2} F$. The econometrician estimating $\hat{\gamma}=\pi F / \operatorname{cov}\left(\Delta C_{F} / C_{F}, \ln R_{F}\right)$ will get a bias [with $D=T / F$ and as $\theta=\pi /\left(\gamma \sigma^{2}\right)$ ] of

$$
\frac{\hat{\gamma}}{\gamma}=D \frac{6 F^{2}}{(F+1)(F+2)} .
$$

Holding $D$ constant, the continuous-time limit corresponds to $F \rightarrow \infty$, and we find the value $\hat{\gamma} / \gamma=6 D$. The discrete-time case where agents would consume at every econometric period corresponds to $F=1$, and then one gets $\hat{\gamma} / \gamma=D$, which can be easily derived directly.

\section{REFERENCES}

Abel, A. (1990). Asset prices under habit formation and catching up with the Joneses. American Economic Review, 80(2):38-42.

Barberis, N., M. Huang, and J. Santos. (2001). Prospect theory and asset prices. Quarterly Journal of Economics 116(1):1-53.

Bernatzi, S., and R. Thaler. (1995). Myopic loss aversion and the equity premium puzzle. Quarterly Journal of Economics 110(1):73-92.

Brav, A., G. M. Constantinides, and C. C. Geczy. (2000). Asset pricing with heterogeneous consumers and limited participation: Empirical evidence. University of Chicago. Mimeo.

Caballero, R. J. (1995). Near-rationality, heterogeneity, and aggregate consumption. Journal of Money, Credit and Banking 27(1):29-48.

Calvo, G. (1983). "Staggered prices in a utility-maximizing framework. Journal of Monetary Economics 12(3):383-398.

Campbell, J. (1999). Asset prices, consumption, and the business cycle. In Handbook of Macroeconomics, J. Taylor and M. Woodford (eds.). Chapter 19, pp. 1231-1303.

- - and J. Cochrane. (1999). By force of habit: A consumption-based explanation of aggregate stock market behavior. Journal of Political Economy 107(2): 205-251.

Cochrane, J. H. (1989). The sensitivity of tests of the intertemporal allocation of consumption to near-rational alternatives. American Economic Review 79: 319-337.

__ and L. Hansen. (1992). Asset pricing explorations for macroeconomics. In 1992 NBER Macroeconomics Annual, O. Blanchard and S. Fischer (eds.). Cambridge, MA: The MIT Press. 
Constantinides, G. M. (1990). Habit formation: A resolution of the equity premium puzzle. Journal of Political Economy 98:519-543.

- J. B. Donaldson, and R. Mehra. (2000). Junior can't borrow: A new perspective on the equity premium puzzle. University of Chicago. Mimeo.

Daniel, K., and D. Marshall. (1997). The equity premium puzzle and the risk-free rate puzzle at long horizons. Macroeconomic Dynamics 1:452-484.

- and - (1999). Consumption-based modeling of long-horizon returns. Kellogg Graduate School of Management, Northwestern University. Mimeo.

Duffie, D., and T. S. Sun. (1990). Transactions costs and portfolio choice in a discrete-continuous-time setting. Journal of Economic Dynamics $\mathcal{E}$ Control 14(1): $35-51$.

Dynan, K., and D. M. Maki. (2000). Does stock market wealth matter for consumption? Federal Reserve Board. Mimeo.

Fischer, S. (1977). Long-term contracts, rational expectations, and the optimal money supply rule. Journal of Political Economy 85(1):191-205.

Gabaix, X., and D. Laibson. (2000a). A boundedly rational decision algorithm. AEA Papers and Proceedings, May, pp. 433-438.

$\longrightarrow$, and $\longrightarrow$. (2000b). Bounded rationality and directed cognition. Harvard University and MIT. Mimeo.

Grossman, S. J., and G. Laroque. (1990). Asset pricing and optimal portfolio choice in the presence of illiquid durable consumption goods. Econometrica 58(1):25-51.

- A. Melino, and R. J. Shiller. (1987). Estimating the continuous time consumption based asset pricing model. Journal of Business and Economic Statistics 5:315-327.

Hall, R. E. (1978). Stochastic implications of the life cycle-permanent income hypothesis: Theory and evidence. Journal of Political Economy 86(6):971-987.

Hansen, L. P., and K. Singleton. (1983). Stochastic consumption, risk aversion, and the temporal behavior of asset returns. Journal of Political Economy 91:249268.

_ , and R. Jagannathan. (1991). Implications of security market data for models of dynamic economics. Journal of Political Economy 99(2):225-262.

He, H., and D. M. Modest. (1995). Market frictions and consumption-based asset pricing. Journal of Political Economy 103:94-117.

Heaton, J., and D. J. Lucas. (1996). Evaluating the effects of incomplete markets on risk sharing and asset pricing. Journal of Political Economy 104(3):443-487.

Kennickell, A. B., M. Starr-McCluer, and B. Surette. (2000). Recent changes in U.S. family finances: Results from the 1998 Survey of Consumer Finances. Federal Reserve Bulletin, January, pp. 1-29.

Kocherlakota, N. R. (1996). The equity premium: It's still a puzzle. Journal of Economic Literature 34(1):42-71.

Lettau, M., and S. Ludvigson (2001). Understanding trend and cycle in asset values: Bulls, bears, and the wealth effect on consumption. Federal Reserve Bank of New York. Mimeo.

Lucas, R. E. (1978). Asset prices in an exchange economy. Econometrica 46:14291446.

Luttmer, E. (1995). Asset pricing in economics with frictions. University of Chicago. Mimeo.

Lynch, A. (1996). Decision frequency and synchronization across agents: Impli- 
cations for aggregate consumption and equity returns. Journal of Finance 51(4):1479-1497.

- , and P. Balduzzi. (2000). Predictability and transaction costs: The impact on rebalancing rules and behavior. Journal of Finance 55(5):2285-2309.

Mankiw, N. G. (1982). Hall's consumption hypothesis and durable goods. Journal of Monetary Economics 10:417-425.

, and S. Zeldes. (1991). The consumption of stockholders and nonstockholders. Journal of Financial Economics 29(1):97-112.

Marshall, D., and N. Parekh. (1999). Can costs of consumption adjustment explain asset pricing puzzles? Journal of Finance 54(2):623-654.

Mehra, R., and E. Prescott. (1985). The equity premium: A puzzle. Journal of Monetary Economics 15:145-161.

Merton, R. (1969). Lifetime portfolio selection under uncertainty: The continuous time case. Review of Economics and Statistics 51:247-257.

Poterba, J. (2000). Stock market wealth and consumption. Journal of Economic Perspectives 14(2):99-118.

Ramsey, F. (1928). A mathematical theory of saving. Economic Journal 38(December):543-559.

Rogers, L.C.G. (2001). The relaxed investor and parameter uncertainty. Finance and Stochastics 5(2):131-154.

Rubinstein, M. (1976). The valuation of uncertain income streams and the pricing of options. The Bell Journal of Economics 7(2):407-425.

Shiller, R. J. (1982). Consumption, asset markets, and macroconomic fluctuations. Carnegie Mellon Conference Series on Public Policy 17:203-238.

Starr-McCluer, M. (2000). Stock market wealth and consumer spending. Federal Reserve Board of Governors. Mimeo.

Taylor, J. (1979). Staggered wage setting in a macro model. American Economic Review 69(2):108-113.

Thaler, R. (1992). Savings, fungibility, and mental accounts. In The Winner's Curse: Paradoxes and Anomalies of Economic Life. Princeton NJ: Princeton University Press, pp. 107-121.

Vissing, A. (2000). Limited stock market participation and the equity premium puzzle. University of Chicago. Mimeo.

\section{Comment}

ANTHONY W. LYNCH

New York University

\section{Introduction}

Gabaix and Laibson extend some earlier work examining the effects of infrequent consumption decision-making by individuals. Grossman and Laroque (1990) developed a continuous-time model in which an individual adjusts consumption infrequently because of proportional adjustment costs. Marshall and Parekh (1999) present numerical results for an economy composed of heterogeneous agents behaving in this way. Cali- 
brating equity returns to U.S. data, they find that undetectably small consumption adjustment costs can alleviate the equity-premium puzzle by delivering the low volatility of aggregate consumption growth and its low correlation with equity return found in U.S. data.

Agents facing proportional adjustment costs use a state-dependent decision rule. As an alternative, Lynch (1996) examined an economy in which decisions are made at fixed intervals and are unsynchronized across agents. Agents choose nondurable consumption and portfolio composition, and either or both can be chosen infrequently. A small utility cost is associated with both decisions being made infrequently. Calibrating returns to the U.S. economy, Lynch (1996) also found that less frequent and unsynchronized decision making delivers the low volatility of aggregate consumption growth and its low correlation with equity return found in U.S. data. Allowing portfolio rebalancing to occur every period has a negligible effect on the joint behavior of aggregate consumption and returns.

Gabaix and Laibson present a continuous-time generalization of Lynch's model and are able to obtain analytic expressions for the bias to risk aversion imparted by less frequent consumption adjustments. The paper also calibrates a version of the model that incorporates temporal aggregation, delayed adjustment, and nonparticipation in stocks by a fraction of the agents. Consistent with the results in Lynch (1996) and Marshall and Parekh (1999), Gabaix and Laibson also find that a delayed-consumption-adjustment model can help explain the equitypremium puzzle by producing lower consumption-growth volatility and lower contemporaneous covariance of consumption growth with equity returns.

Although not modeled explicitly by Lynch or by Gabaix and Laibson, constant decision intervals arise when it is costly to gather information about wealth innovations and to solve optimization problems. Duffie and Sun (1990) presented a model of this type and showed that if utility is power, risky-asset return is in geometric Brownian motion, and transaction costs are proportional to wealth, then the optimal decision interval is a constant.

This discussion first describes the model and summarizes some of its key implications. Then the calibration and empirical work are discussed. Finally, some general comments and conclusions are presented.

\section{Model Setup and Main Results}

The economy has a riskless rate $r$ and a risky return that follows geometric Brownian motion with an instantaneous mean return of $\pi+r$ and an 
instantaneous variance of $\sigma^{2}$. Agents have power utility and adjust consumption every $D$ periods. At each adjustment time, agents set aside an amount for consumption over the next $D$ periods, which earns the riskless rate $r$ until consumed. The agents place their remaining wealth in an investment portfolio that is continuously rebalanced. Thus, the optimal risky-asset weight $\theta$ is same as in the $D=0$ case: $\theta=\pi /\left(\gamma \sigma^{2}\right)$. The economy has a continuum of agents, indexed by adjustment times, which are uniformly distributed over any interval of length $D$.

When $D=0$, the econometrician still faces temporal aggregation. The variance of log per-period aggregate consumption growth is $\frac{2}{3}$ of instantaneous volatility when $D=0$, is declining in $D$, and is approximately $1 / D$ times instantaneous volatility when $D$ is large. Instantaneous log consumption-growth autocorrelation is 0 at all lags when $D=0$. In contrast, because of temporal aggregation, log per-period consumption growth autocorrelation when $D=0$ is $\frac{1}{4}$ at lag 1 and is 0 at lags of 2 or more. With temporal aggregation and $D>0$, log per-period consumption growth autocorrelation is positive and decreasing in lag length at lags less than $D+2$ and is 0 at lags of $D+2$ or more. The instantaneous contemporaneous covariance of log consumption growth with log riskyasset return with $D=\mathrm{O}$ is $\theta \sigma^{2}$. The contemporaneous covariance of log per-period consumption growth with log per-period risky-asset return is $\theta \sigma^{2} / 2$ with $D=0$ and is $\theta \sigma^{2} /(6 D)$ with $D>1$. Finally, with $D=0$, the instantaneous covariance of log consumption growth with lagged log risky-asset return is 0 at all lags $>0$, while the covariance of log perperiod consumption growth with lagged log per-period risky-asset return is positive at lags less than 2 and is 0 at lags of 2 or more. Once $D>$ 1 , the covariance of log per-period consumption growth with lagged log per-period risky-asset return is positive at lags less than $D+2$ and is 0 at lags of $D+2$ or more.

To summarize, the contemporaneous covariance of consumption growth with risky-asset return is lower than instantaneous due to temporal aggregation alone, lower still due to infrequent adjustment, and decreasing in $D$. The variance of consumption growth is lower than the instantaneous variance due to temporal aggregation alone, is lower still due to infrequent adjustment, and is decreasing in $D$. The covariance of consumption growth with lagged consumption growth and with lagged risky-asset return is positive for lags less than 2 due to temporal aggregation alone, and in general is positive for lags less than $D+2$ and zero otherwise.

While the paper typically fixes the lag or the period length and varies $D$, it would be useful to examine what happens to the various statistics 
of interest as the lag or the period length is varied for fixed $D$. Such an analysis would be helpful for generating testable implications, since one particular distribution for $D$ holds empirically.

The paper's assumption that agents continuously rebalance their investment portfolios seems inconsistent with a fixed adjustment interval, since the assumed fixed interval between consumption adjustments is difficult to justify when agents know the risky-asset return. The paper's closed-form solutions rely on continuous portfolio rebalancing by agents. Restricting the ability of an agent to rebalance her portfolio within her adjustment period may affect the distribution of aggregate consumption growth. In an economy with infinite-lived agents making unsynchronized consumption and portfolio decisions every $D$ periods, the crosssectional distribution of agent wealth (expressed as a fraction of total wealth) becomes increasingly disperse over time, indicating that aggregate consumption growth does not have a steady-state distribution. This concern prompted Lynch (1996) to build an overlapping-generations economy with finite-lived individuals and a deterministically growing wealth endowment for each period's newborn. Lynch finds that the implications for aggregate consumption growth of infrequent consumption adjustments are largely unaffected by the portfolio-rebalancing frequency of agents.

\section{Calibration and Empirical Work}

Gabaix and Laibson estimate $D$ based on a cost of adjusting consumption of $0.04 \%$ of wealth and obtain an estimate of 2 years. This estimate is likely to overstate $D$, since their calculations assume continuous portfolio rebalancing. The paper then calibrates a simple macro model with shareholders who delay consumption and nonshareholders who do not delay. The calibration makes many simplifying assumptions: both groups have the same propensity to consume, and the wealth of the two groups is assumed to be uncorrelated. Some sensitivity analysis would be useful.

While the endogenous adjustment period is calculated to be 2 years, the calibrated model has a continuum of agents and $D$ is uniformly distributed from 0 to 30 years. This switch is not innocuous. For example, the empirical section attempts to explain the pattern of covariances between equity $R_{t+1}$ and $\ln \left(C_{t+h} / C_{t}\right)$ as a function of $h$. The paper uses quarterly data for this section and finds these covariances are roughly increasing in $h$ out to at least 20 quarters, particularly for countries with large stock markets. This model generates the upward-sloping pattern out to at least 20 quarters that is found in the data. But this result 
depends critically on $D$ taking values out to at least 20 quarters. If the paper used the calibrated adjustment period of $D=8$ quarters, the covariance pattern would be flat for $h \geq 10$ quarters.

In the calibration section, the paper only compares the model with the data on consumption growth's volatility, contemporaneous covariance with return, and first-lag autocorrelation. Then, the empirical section attempts to explain the pattern of covariances between equity $R_{t+1}$ and $\ln \left(C_{t+h} / C_{t}\right)$ and the pattern of $h$-period Euler equations, both as functions of $h$. However, the distinction between the paper's calibration and empirical work seems artificial. Since the model also provides many other moments that could be compared with the data, it would be useful to calibrate a model and then examine how it performs with respect to a wide range of moments for aggregate consumption growth. A more systematic analysis would be instructive. It would also be useful to have standard errors for the data estimates as part of such an analysis. One approach simulates samples using the model and calculates a distribution for the statistic of interest. This distribution can then be used to calculate a $p$ value for the data estimate under the null that the model holds.

For example, the paper does not examine consumption-growth autocorrelations beyond the first lag, even though the model provides predictions about them. Heaton (1993) finds negative autocorrelation at the 5th lag for quarterly seasonally adjusted consumption changes, negative autocorrelation at the 1st and 4th lags for monthly seasonally adjusted consumption changes, and negative autocorrelation at the 1st, $2 \mathrm{nd}$, and 5 th lags for quarterly non-seasonally-adjusted consumption changes. Negative autocorrelation at any lag is inconsistent with the model. So despite the likely role being played by measurement error, Heaton's results are challenges for the paper's delayed-consumption-adjustment model. Thus, while a useful first step, the empirical work is far from conclusive, and more needs to be done to ascertain whether delayed consumption adjustment is playing a role in the U.S. economy and in other economies.

\section{General Comments and Conclusions}

The assumption of a predetermined delay interval $D$ is difficult to justify. At the very least, agents are likely to adjust consumption after large changes in equity value. While this effect causes all investors to adjust at the same time (see, for example, Marshall and Parekh, 1999), Gabaix and Laibson find the upward bias to risk aversion can still be large. However, their adjustment trigger of $25 \%$ monthly return in absolute value is quite extreme, and the upward bias associated with a more modest and reason- 
able trigger point is likely to be much smaller. A model in which investors adjust consumption after a large change in equity value is empirically distinguishable from the fixed-decision-interval model. The former predicts that the standard Euler equation should perform well in those periods that are preceded by a period with a large equity return in absolute value. Empirical work is needed to characterize the delayedadjustment rule (if any) being used by agents in the U.S. economy and other economies.

The model assumes that equity value is in geometric Brownian motion. There is much evidence that equity returns are predictable and heteroscedastic. The implication may be a delay interval $D$ that depends on the same variables that forecast means and variances.

It seems unlikely that agents always take recent wealth innovations into account when making high-frequency consumption decisions. Gabaix and Laibson's analytical results serve to emphasize the potentially large effect of such behavior on the joint distribution of aggregate consumption and equity return. Hopefully, their work will prompt more theoretical and especially empirical work directed toward understanding how agents delay adjusting their consumption and how this delay affects aggregate consumption in the U.S. and other countries.

\section{REFERENCES}

Duffie, D., and T. Sun. 1990. Transaction costs and portfolio choice in a discretecontinuous-time setting. Journal of Economic Dynamics and Control 14:35-51.

Grossman, S., and G. Laroque. 1990. Asset pricing and optimal portfolio choice in the presence of illiquid durable consumption goods. Econometrica 58:25-51.

Heaton, J. 1993. The interaction of time-nonseparable preferences and time aggregation. Econometrica 61(2):353-385.

Lynch, A.W. 1996. Decision frequency and synchronization across agents: Implications for aggregate consumption and equity return. Journal of Finance 51:14791498.

Marshall, D.A., and N.G. Parekh. 1999. Can costs of consumption adjustment explain asset pricing puzzles? Journal of Finance 54:623-654.

\section{Comment}

MONIKA PIAZZESI

UCLA and NBER

\section{Introduction}

An economy populated by a representative agent with power utility predicts an equity premium which is far below the realized equity pre- 
mium in postwar data, at least for "reasonable parameters" for the endowment process and the coefficient of relative risk aversion $\gamma$. This is the equity-premium puzzle stated by Mehra and Prescott (1985). Simply increasing $\gamma$ (and somehow arguing that this is "reasonable") does not solve the puzzle, because a high $\gamma$ counterfactually leads to a high riskfree rate. The few models in the literature today that may be considered puzzle-free still rely on high $\gamma^{\prime}$ s. An example is Campbell and Cochrane (1999), who use an average $\gamma$ of 50. An argument that relies on estimation bias for $\gamma$ alone, as suggested by the title of the paper, cannot therefore be enough to reconcile the standard model with the data. But there is more to the model of Gabaix and Laibson than the title indicates, because it is populated by agents whose heterogeneity matters.

The high equity premium and the low risk-free rate are, literally speaking, no puzzles in the model: asset prices are specified exogenously. The endogenous variables in this model are the consumption processes of individual investors. By summing these over a group of investors, the paper obtains a measure of aggregate consumption. The interpretation of this portfolio choice model, or Merton model, as a production economy with exogenous production technologies (or as a small open economy) leads to another endogenous variable: net borrowing by this group of investors (or the current account). The behavior of these endogenous variables (consumption and net borrowing) is what is puzzling in models with exogenous returns (such as Constantinides, 1990). My discussion will thus concentrate on the model-implied behavior of these endogenous variables.

The model is a continuous-time version of Lynch (1996). Agents are indexed by a first adjustment time $i$ and an interval length $D_{i}$ between adjustments, which together define an (exogenous) adjustment sequence $\left\{i, i+D_{i}, i+2 D_{i}, \ldots\right\}$. Between adjustment times $\left[i+j D_{i}, i+(j\right.$ $\left.+1) D_{i}\right), j \in N$, agents do not know the returns of risky assets and do not trade them. This feature makes assets illiquid. As in a limitedparticipation model, the Euler equations for only a subset of agents hold at any point in time $t$ in this economy. With adjustment delays, the firstorder conditions for risky-asset holdings at time $t$ are only satisfied for those agents that are adjusting at time $t$. The intuition from a closedeconomy version of this model tells us that in this case agents need to be compensated to hold these illiquid assets. The resulting equity premium is not so much a risk premium in the usual sense as a liquidity premium.

We need to be careful, however, in applying closed-economy intuition to this setup, because it is not clear whether the implications of the model will survive in a closed-economy setting. The reason is that agents in the model continuously observe the riskless rate, which is 
assumed to be constant. In a closed economy, the riskless rate responds to stock-market movements and therefore reveals information from other agents in the economy (who get to adjust their consumption earlier in response to these movements). This means that even agents who do not directly observe stock returns can infer from the riskless rate whether the stock market just tanked and thus can adjust their consumption immediately. The closed-economy version of the model with learning will be more difficult to solve, but future research will hopefully tell us how it behaves.

The puzzles lie in the numbers, so I will compare the model's implication with the joint time series of quarterly U.S. aggregate consumption and real stock returns. I will show that adjustment delays alone cannot provide an explanation for the equity premium. The model fails along three main dimensions: (i) consumption growth from the model is too autocorrelated, (ii) the normalized covariance of returns with consumption monotonically increases with horizon in the model, while it is hump-shaped in the data with a peak at 2 years, and (iii) returns are assumed to be i.i.d., while they are predictable in the data.

The reason for (i) is that stock-market shocks trigger a series of individual consumption adjustments in the same direction by agents who only get to adjust later to the shock. The resulting aggregate consumption growth process thus looks autocorrelated and predictable by stock returns. The model does not seem to generate too much predictability for consumption, but it does imply too much autocorrelation for consumption growth.

The reason for (ii) is that as we lower the frequency at which we observe data relative to the frequency at which consumption decisions are made, the model looks more and more like a standard model without adjustment delays. In standard models the covariance between consumption growth and stock returns divided by horizon increases with horizon. This feature is counterfactual; it is known as the equity-premium puzzle at long horizons and is documented by Cochrane and Hansen (1992).

There is a long list of variables that successfully predict stock returns in (iii). The list includes term spreads (Campbell, 1987), the dividendearnings ratio (Lamont, 1996), and the consumption/wealth ratio (Lettau and Ludvigson, 2001). I show that even lagged consumption growth (which is a variable directly taken from the model) is a predictor (but of course less successful than other variables).

In addition to these three problems, the model may be relying on large and counterfactual net borrowing from "foreigners" (agents whose consumption is not used to define aggregate consumption) to sustain the 
exogenously fixed low risk-free rate, but I have not looked at the behavior of net borrowing.

In the process of documenting the properties of the model, I also show that the first three autocorrelations of consumption growth are significantly different from zero in the data. Moreover, consumption growth is heteroscedastic in the data. For example, a $\operatorname{Garch}(1,1)$ is significant. These two properties mean that consumption growth is certainly not i.i.d., an assumption often made by recent consumption-based asset pricing models (following Hall, 1978). Heteroscedasticity may be important for explaining the time variation in expected returns which is not captured in this paper. Models that replicate this time variation typically rely on features of preferences which produce time-varying risk aversion (Campbell and Cochrane, 1999; Barberis, Huang, and Santos, 2001; Veronesi, 2001).

I also show that the cross-correlation of consumption growth and stock returns data seems to be seasonal. This seasonality appears even though the consumption data are seasonally adjusted. At first sight this adjustment looks successful, because the autocorrelation function of consumption growth does not show any obvious seasonal patterns. The cross-moments with returns, however, seem to indicate that it may matter for stock pricing that real-life investors are consuming a seasonal consumption process. This raises the question whether the predictability of consumption growth is a feature of the data that should be matched by an asset-pricing model.

The following discussion will thus concentrate on the autocorrelation and predictability of consumption growth, the predictability of returns, and the equity premium at long horizons. Here, "consumption" always refers to aggregate consumption. I will then return to the interpretation of adjustment delays in terms of cognitive costs that is offered in this paper and suggest extensions.

\section{Data and Calibration}

The comparison of the model with the data relies on two series: consumption and real stock returns. Consumption is for nondurables and services excluding shoes and clothing, seasonally adjusted in 1996 chainweighted dollars. The returns are for all stocks traded on NASDAQ, AMEX, and the NYSE. The calculation of real returns relies on the consumer price index. The sample consists of quarterly data from 1953:1 to 2000:3.

Since I use different consumption and returns data than the paper, I also use slightly different parameter values to calibrate the model: $r+\pi$ 
$=0.08, \sigma=0.16, \gamma=4, D_{i}=4$ or $D_{i}=10, \forall i$. I assume that initial adjustment times $i$ are uniformly distributed over $[0, D]$.

\section{Autocorrelation of Consumption Growth}

Figure 1 shows the autocorrelation of consumption growth at different lags $h$ together with $95 \%$ confidence bounds. The autocorrelation is significant up to the third quarter, which means that consumption growth is definitely not i.i.d. The figure also shows the autocorrelations implied by the model for $D=4$ and $D=10$. The general pattern is that the autocorrelation in a model with interval length $D$ between two decisions dies off after $D$ periods. The autocorrelation in the data seems to be best matched by choosing $D=4$. The first two autocorrelations of 0.85 and 0.57 produced by the model for $D=4$ are clearly too high compared to the data.

As an aside, I would like to add that autocorrelation is not the only dimension in which consumption growth is not i.i.d. Consumption

\section{FIGURE 1 AUTOCORRELATION OF CONSUMPTION GROWTH}

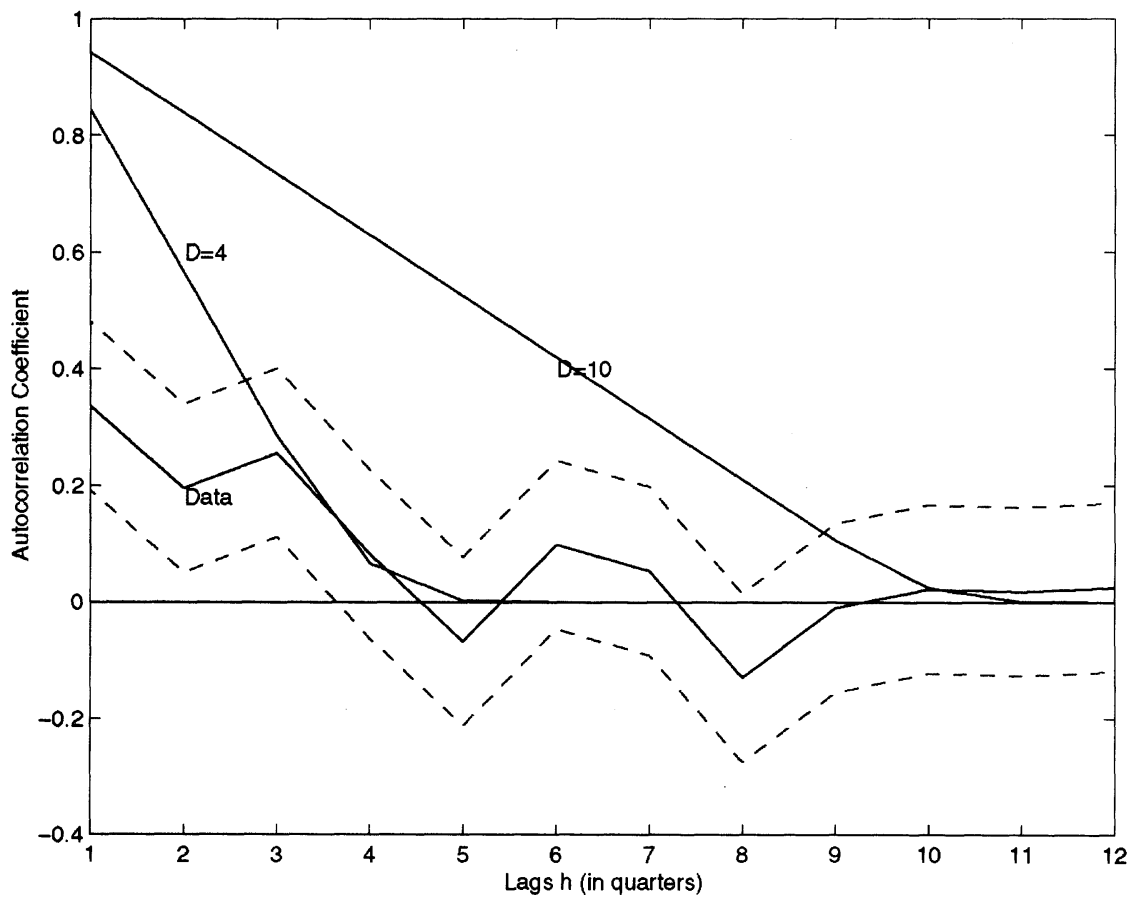


Table 1 MAXIMUM-LIKELIHOOD ESTIMATES OF $\Delta \log c_{t}=c_{0}+c_{1} \Delta \log c_{t-1}$ $+c_{2} \Delta \log c_{t-2}+c_{3} \Delta \log c_{t-3}+\varepsilon_{t}$

\begin{tabular}{ccccccc}
\hline$c_{0}$ & $c_{1}$ & $c_{2}$ & $c_{3}$ & $\alpha_{0}$ & $\alpha_{1}$ & $\alpha_{2}$ \\
\hline 0.01 & 0.31 & 0.03 & 0.23 & 0.00 & 0.03 & 0.95 \\
$(7.04)$ & $(4.01)$ & $(0.33)$ & $(2.93)$ & $(0.64)$ & $(1.19)$ & $(29.56)$ \\
\hline
\end{tabular}

Here $\varepsilon_{t}$ is conditionally normal with mean 0 and variance $\sigma_{t}^{2}=\alpha_{0}+\alpha_{1} \varepsilon_{t-1}^{2}+\alpha_{2} \sigma_{t-1}^{2}$. The estimation uses quarterly data on U.S. consumption of nondurables and services without shoes and clothing from 1953:1 to 2000:3. $t$-statistics are in brackets.

growth is also heteroscedastic, a property which may be important for explaining the time variation in expected returns (which is not captured by the model). This can be seen from Table 1 , which reports the maximum-likelihood estimates of an AR(3) combined with a Garch $(1,1)$. The estimate of the Garch parameter $\alpha_{2}$ is 0.95 and is strongly significant. The autoregressive parameters are partial correlations, so they differ from Figure 1, which shows autocorrelations.

\section{Equity Premium at Long Horizons}

To see how the model behaves as we vary the observation horizon $h$ for a fixed decision interval length $D \geq 1$, consider the following equation that determines the equity premium in the model:

$\pi=\frac{\operatorname{cov}\left(\log \left(c_{t+h+1} / c_{t}\right), \log R_{t, t+h}\right)}{h} \cdot \gamma \cdot 6 D$

Figure 2 shows the covariance factor on the right-hand side of this equation, the covariance of consumption growth and stock returns divided by the horizon. In the data, this covariance is hump-shaped as a function of horizon: increasing up to 2 years and then decreasing. The model predicts a monotonically increasing covariance. The reason is that as we lower the observation frequency relative to the decision interval length $D$, the model behaves more and more like the original Merton model without adjustment delays. Therefore the model predicts a high covariance of consumption growth and stock returns at long horizons, which is counterfactual.

The equity premium at long horizons was noted by Cochrane and Hansen (1992) and was seen as causing a problem for the timeaggregation literature because aggregation problems matter less as we lower the frequency at which we observe the data. The same now applies to a model with adjustment delays. Figure 2 does not show the 
FIGURE 2 COVARIANCE OF $\log \left(c_{t+h} / c_{t}\right)$ AND $\log R_{t, t+h}$ DIVIDED BY $h$

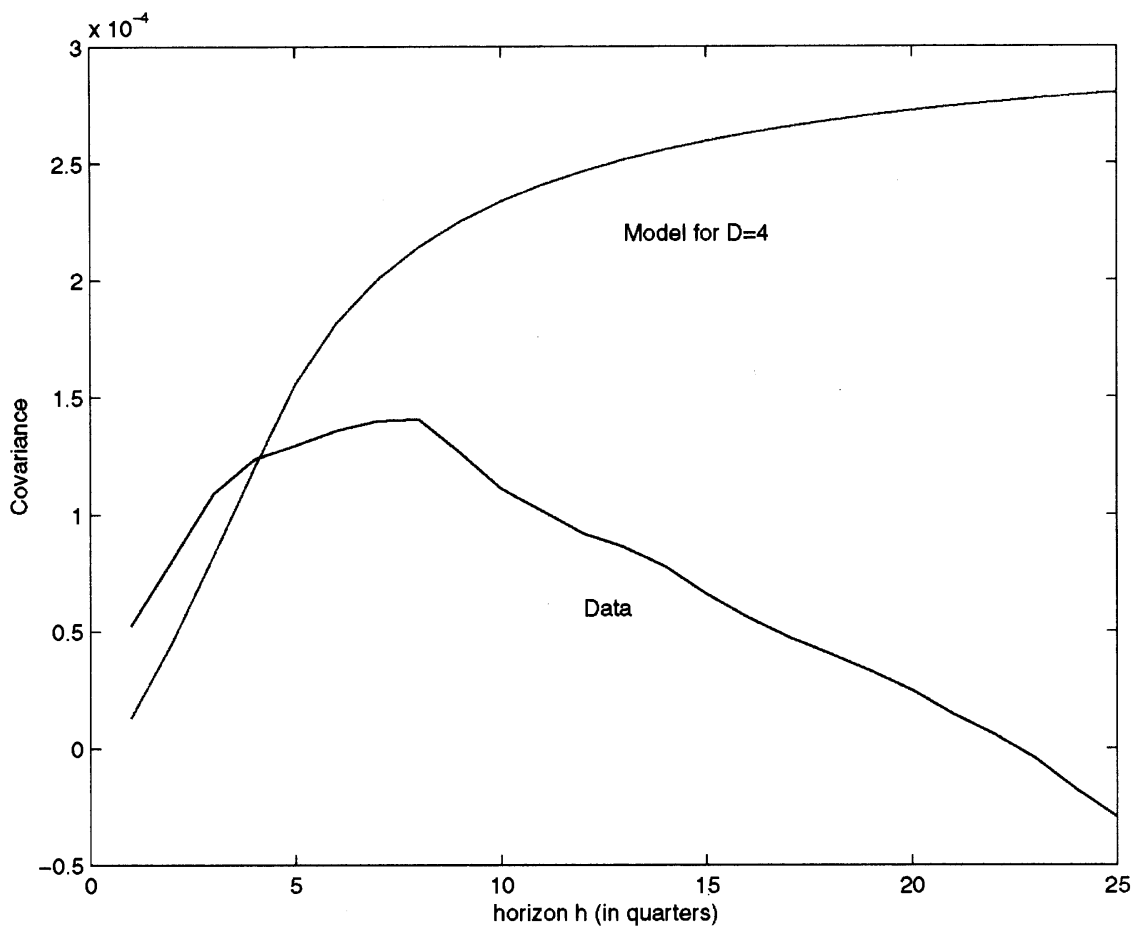

standard errors around the covariance estimates, which get large with horizon to the extent that the hump in the empirical covariance is not significant. The equity premium, however, is not much of a puzzle if we take into account standard errors in this case (as can be seen from the cross-correlation at $h=0$ in Figure 5 below).

\section{Predictability of Consumption Growth}

To look at the predictability of consumption with stock returns, Gabaix and Laibson compute the cumulative covariance of log stock returns log $R_{t, t+1}$ from time $t$ to time $t+1$ with consumption growth $\log \left(c_{t+1+h} / c_{t}\right)$ from time $t$ to time $t+1+h$, for different quarterly horizons $h$. By decomposing this covariance measure into its individual elements, we get

$\operatorname{cov}\left(\log \frac{c_{t+h+1}}{c_{t}}, \log R_{t, t+1}\right)=\sum_{i=0}^{h} \operatorname{cov}\left(\log \frac{c_{t+i+1}}{c_{t+i}}, \log R_{t, t+1}\right)$. 
From the last equation, we can see that this cumulative covariance measure does not only reflect whether stock returns predict consumption growth, because part of the covariance is due to the contemporaneous covariance $\operatorname{cov}\left(\log \left(c_{t+1} / c_{t}\right), \log R_{t, t+1}\right)$ between returns and consumption growth.

Figure 3 plots this cumulative consumption measure (like Figure 7 in the paper), while Figure 4 plots the individual components in the sum on the right-hand side of the last equation. Both figures are based on U.S. data for nondurables and services instead of the total consumption series from different countries used in the paper. The dashed lines are $95 \%$ confidence bounds based on Newey-West standard errors. Figure 3 shows that the contemporanous covariance estimate in the data is already nonzero, and then the covariance measure increases up to 7 quarters. Beyond that, the covariance slightly decreases with horizon, but confidence bounds become large. The figure shows that the covariance pattern in the data is well replicated by the model if the interval length $D$ between decisions is set to 4 quarters.

FIGURE 3 COVARIANCE OF $\log \left(c_{t+1+h} / c_{t}\right)$ AND $\log R_{t, t+1}$

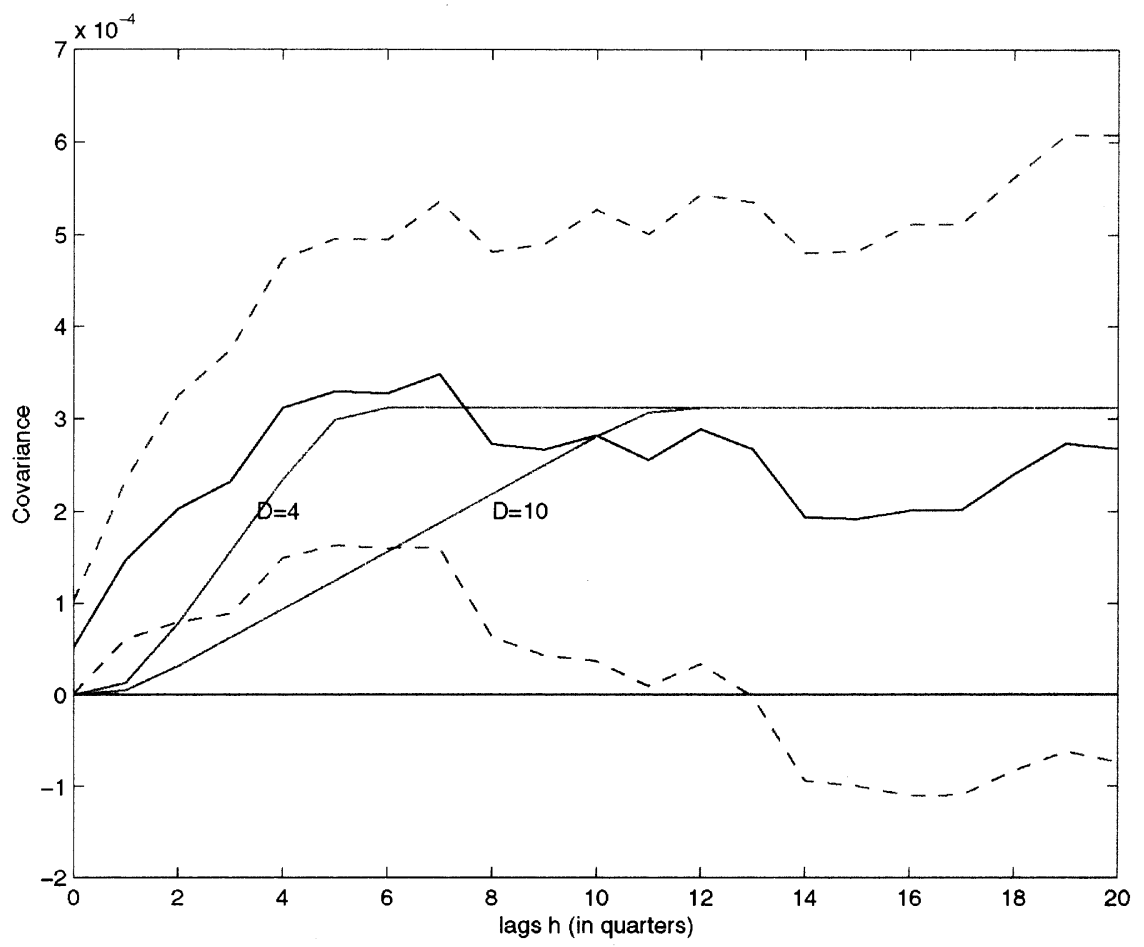


FIGURE 4 COVARIANCE OF $\left(c_{t+1+h} / c_{t+h}\right)$ AND $\log R_{t, t+1}$



The covariance of the total consumption data (used in the paper) with returns seems to increase with horizon. To replicate this, Gabaix and Laibson use a distribution for $D$ over $[0,30]$ years to compute the covariance measure from the model. This is not necessary for data on nondurables and services. Section 6.2 of the paper compares Figure 7 with the plain Merton model with i.i.d. consumption growth. This is not really an appropriate comparison, because it is clear that a model where consumption growth is assumed to be i.i.d. does not imply any predictability. Models with exogenous returns like Constantinides (1990) tend to produce too much predictability, and so they provide a more natural benchmark.

The individual covariances in Figure 4 represent the slope of the cumulative covariance function in Figure 3 . We can see that the slope is significant and positive for horizons 1,2 , and 4 , while it becomes negative at horizon 8 . This pattern looks somewhat seasonal, even though the consumption series is seasonally adjusted. This pattern suggests that the covariance increase until $h=7$ in Figure 3 may be due to seasonalities. In 
this case, it is not clear whether this predictability is a feature that the model should match. More generally, the pattern raises doubts about the use of seasonally adjusted data for tests of consumption-based asset pricing models.

\section{Predictability of Returns}

Stock returns can be predicted with a large number of variables. Figure 5 shows the cross-correlation between current consumption growth $\log \left(c_{t+1} / c_{t}\right)$ and returns from time $t+h$ to $t+h+1$ for varying horizons $h$ together with approximate $95 \%$ confidence bounds (computed as $\pm 2 \sqrt{T}$, where $T$ is the number of observations in the sample). The pattern of this cross-correlation for $h=0,-1,-2,-4,-8$ shows again that the equity premium is measured with a lot of noise (supposing the standard Euler equation holds) and that consumption growth is predictable with stock returns as documented in Section 5. The interesting stylized fact that emerges from this graph is that the cross-correlation is

FIGURE 5 CROSS-CORRELATION OF $\log \left(c_{t+1} / c_{t}\right)$ WITH $\log R_{t, t+1+h}$

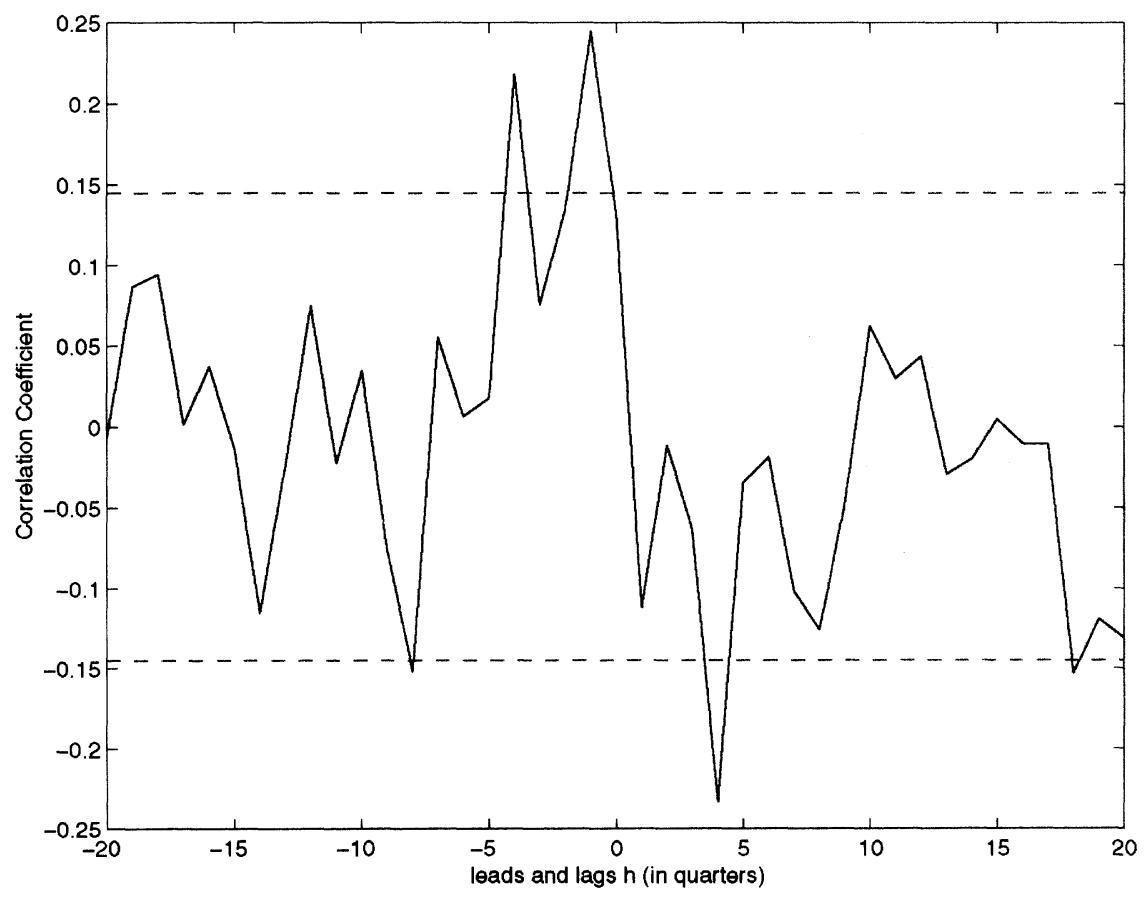


also significant at $h=4$. This means we can use current returns to predict consumption growth one year from now.

The model by Gabaix and Laibson is not consistent with this feature of the data, because it assumes that these returns are i.i.d. and thus not predictable. Future research will hopefully show whether adjustment delays can be combined with something else, such as habit formation, so that the extended model can capture this important stylized fact.

\section{Some Evidence about Cognitive Costs}

The paper assumes that agents do not receive or process stock-market information between any two periods. If this assumption is a good description of individual behavior, real net mutual-fund inflows should react to past stock return information. To check this implication of the model, I collect monthly data on net inflows into stock funds from 1984:1 to 2001:2. These data can be obtained from the Web site of the Investment Company Institute. Real inflows are computed based on the consumer price index. I also subtract a linear trend from the real inflows. Figure 6 shows that only

FIGURE 6 CORRELATION BETWEEN Inflows(t) AND $\log R_{t-h, t-h+1}$

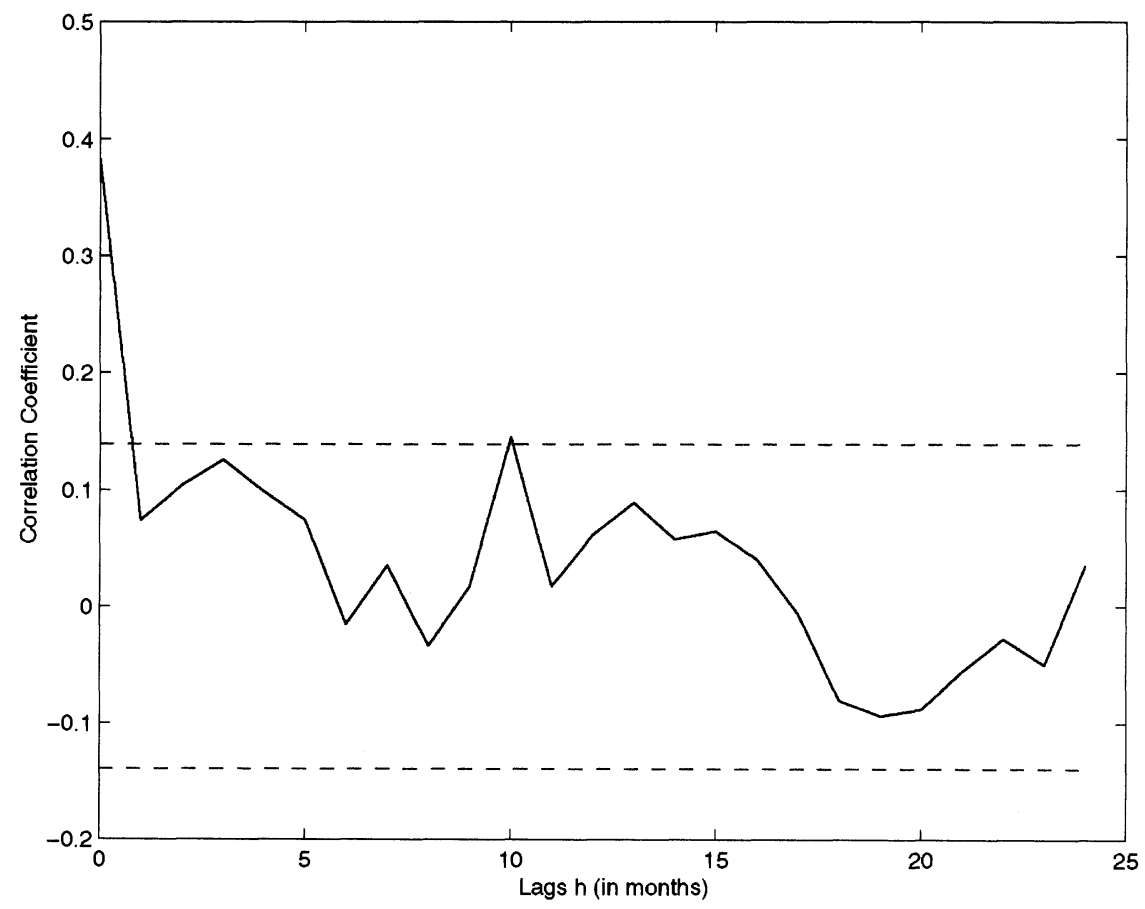


the contemporaneous correlation between real net inflows and returns is significant, not the correlation between inflows and past returns. While this is certainly not conclusive evidence against cognitive costs, the graph still provides some evidence that investors do not react to past return information when choosing their portfolio.

\section{Extension to General Equilibrium}

The riskless rate is exogenous in this model and therefore does not reveal any information that agents have who have only recently adjusted their portfolio. I doubt this feature of the model will still be true in a closed-economy version where the riskless rate is allowed to move in response to a stock-market crash. This version is not easy to compute, but the wealth distribution matters even without idiosyncratic shocks. It would be interesting to link it to models in the incomplete-market literature (e.g., Krusell and Smith, 1997) which also try to increase individual consumption volatility like Gabaix and Laibson, but with a different mechanism. There is some hope that a combination of the two will be successful at explaining the equity-premium puzzle.

\section{REFERENCES}

Barberis, N., M. Huang, and T. Santos. (2001). Prospect theory and asset prices. Quarterly Journal of Economics, February, 116, 1-54.

Campbell, J. Y. (1987). Stock returns and the term structure. Journal of Financial Economics 18:373-399.

- and J. H. Cochrane. (1999). By force of habit: A consumption-based explanation of aggregate stock market behavior. Journal of Political Economy 107:205-251.

Cochrane, J. H., and L. P. Hansen. (1992). Asset pricing lessons for macroeconomics. In 1992 NBER Macroeconomics Annual, O. Blanchard and S. Fischer (eds.). Cambridge, MA: The MIT Press, pp. 115-1165.

Constantinides, G. M. (1990). "Habit formation: A resolution of the equity premium puzzle." Journal of Political Economy 98:519-543.

Hall, R. (1978). Stochastic implications of the life cycle-permanent income hypothesis: Theory and evidence. Journal of Political Economy 86:971-987.

Krusell, P., and A. Smith. (1997). Income and wealth heterogeneity, portfolio choice, and equilibrium asset returns. Macroeconomic Dynamics 1(2):245-272.

Lamont, O. (1996). Earnings and expected returns. Cambridge, MA: National Bureau of Economic Research. NBER Working Paper 5671.

Lettau, M., and S. Ludvigson. (2001). Resurrecting the (C)CAPM: A crosssectional test when risk premia are time-varying. Journal of Political Economy, forthcoming.

Lynch, A. (1996). Decision frequency and synchronization across agents: Implications for aggregate consumption and equity return. Journal of Finance 51:14791498. 
Mehra, R. and E. Prescott. (1985). The equity premium puzzle. Journal of Monetary Economics 15:145-161.

Veronesi, P. (2001). Belief-dependent utilities, aversion to state uncertainty and asset prices. Chicago Graduate School of Business. Working Paper.

\section{Discussion}

David Laibson admitted that how to fix $D$, the length of the period between readjustments of consumption, is an important question, and that one would not expect everyone in the economy to have the same $D$. He explained that the assumption of continuous rebalancing was not a crucial one, as it affected only second-order terms. He was very receptive to the idea that some important financial events capture people's attention. An extension to the model to capture this phenomenon through a Poisson arrival rate of important events affects the results only slightly. Laibson recognized that dealing with the long-horizon evidence was important and suggested that the picture would look better using international data. He also said that at long horizons, standard errors become very large, so the evidence neither supported nor rejected the framework.

Robert Barsky suggested that if consumption had to be committed in advance, the effects could be the same as when investors rebalance their portfolios only intermittently because of cognitive costs. He asked whether the model could deal with the puzzle that stocks outperform bonds over long periods. Laibson agreed that cognitive costs are just one possible explanation for delayed adjustment. He guessed that the model had nothing to say about the returns on stocks relative to bonds.

David Romer suggested that the authors should look more carefully at the equity premium over long rather than short horizons, as their explanation seemed to have an effect only at short horizons. He commented that even if the model failed to explain all of the puzzle at long horizons, it was still a useful contribution. He did not see why there should be one single explanation for the entire equity-premium puzzle, a view with which Laibson was sympathetic. Romer also said that the fact that the equity premium had fallen in recent years made him nervous about theories that predict a premium at all times and places. Xavier Gabaix remarked that, according to recent surveys, it appears that the public's expected return on stocks remains high, even though actual returns have fallen.

Nobuhiro Kiyotaki suggested that limited participation can arise endogenously from the costs of rebalancing portfolios. He suggested that 
the authors could get a sense of the importance of cognitive costs by looking at the size of asset holdings of participants and nonparticipants in the stock market.

Greg Mankiw was struck by the fact that the model predicted positive autocorrelation of consumption growth, counter to some of the empirical evidence. Laibson responded that he thought the model did reasonably well on this score. Jim Stock said he would like to see an examination of the temporal aggregation problem in this context.

Gertler suggested that looking at the standard deviation of individual consumption in the model and in the data would be a good way of evaluating the empirical plausibility of the model. Laibson replied that he believed the jumps in consumption predicted by the model were of a reasonable order of magnitude. 